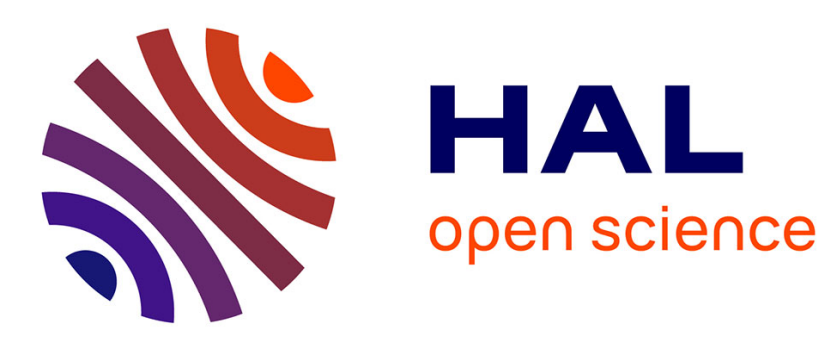

\title{
System identification of microwave filters from multiplexers by rational interpolation
}

Sanda Lefteriu, Martine Olivi, Fabien Seyfert, Matteo Oldoni

\section{To cite this version:}

Sanda Lefteriu, Martine Olivi, Fabien Seyfert, Matteo Oldoni. System identification of microwave filters from multiplexers by rational interpolation. Automatica, 2016. hal-01357934

\section{HAL Id: hal-01357934 \\ https://inria.hal.science/hal-01357934}

Submitted on 30 Aug 2016

HAL is a multi-disciplinary open access archive for the deposit and dissemination of scientific research documents, whether they are published or not. The documents may come from teaching and research institutions in France or abroad, or from public or private research centers.
L'archive ouverte pluridisciplinaire HAL, est destinée au dépôt et à la diffusion de documents scientifiques de niveau recherche, publiés ou non, émanant des établissements d'enseignement et de recherche français ou étrangers, des laboratoires publics ou privés. 


\title{
System identification of microwave filters from multiplexers by rational interpolation
}

\author{
Sanda Lefteriu ${ }^{a}$, Martine Olivi ${ }^{b}$, Fabien Seyfert $^{\mathrm{b}}$, Matteo Oldoni $^{\mathrm{c}}$

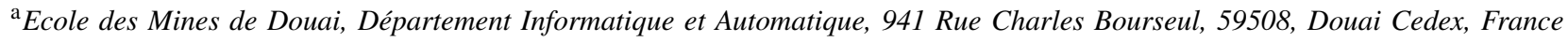 \\ bINRIA, BP 93, 06902 Sophia-Antipolis Cedex, France
}

' SIAE Microelettronica Spa, 21, via Buonarroti, 20093 Cologno Monzese, Italy

\begin{abstract}
Microwave multiplexers are multi-port structures composed of several two-port filters connected to a common junction. This paper addresses the de-embedding problem, in which the goal is to determine the filtering components given the measured scattering parameters of the overall multiplexer at several frequencies. Due to structural properties, the transmission zeros of the filters play a crucial role in this problem, and, consequently, in our approach. We propose a system identification algorithm for deriving a rational model of the filters' scattering matrix. The approach is based on rational interpolation with derivative constraints, with the interpolation conditions being located precisely at the filters' transmission zeros.
\end{abstract}

Key words: System identification; Interpolation; Continuous time filters; Scattering parameters; System transfer functions.

\section{Introduction and motivation}

Microwave multiplexers are present in nearly every transmission or reception unit of communication systems. They are composed of two-port filter structures (Fig. 1) connected to a common support, referred to as a junction (Fig. 3). Consequently, the multiplexer is a multi-port system with a large number of inputs and outputs. The practical realization of such devices is difficult because the computer simulated characteristics of the multiplexer, previously obtained from the design specifications, cannot be manufactured exactly. Therefore, filters are equipped with screws which can be tuned in the final realization phase to match the desired specifications. Tuning is a time consuming operation for microwave engineers in terms of person-hours, so algorithms aimed at solving this problem would offer great benefits. While tuning a multiplexer, it is often not possible to detach the filters because the multiplexer has been manufactured in one piece, or because the repeated plugging and unplugging may lead to defects. As a result, developing tuning techniques for multiplexers which rely solely on external measurements, in particular on frequency domain scattering measurements, is an important research problem.

Email addresses: sanda. lefteriu@mines-douai.fr (Sanda Lefteriu), martine.olivi@inria.fr (Martine Olivi), fabien.seyfert@inria.fr (Fabien Seyfert), matteo.oldoniesiaemic.com (Matteo Oldoni).
We refer to the problem of determining a rational description of the filters composing a multiplexer as the de-embedding problem and its statement is the following: Given multiport scattering measurements of the multi-port multiplexer structure for several frequencies, we wish to derive the scattering matrix of each reciprocal filter composing the structure.

Methods currently available for de-embedding rely on neural networks [22] or on the minimization of a tuning criterion [29]. However, these gradient algorithms might suffer from the issue of reaching a local minimum of their tuning criterion rather than the desired global one. Moreover, these optimization-based techniques give no real insight into the internal physical state of the multiplexer.

Our approach is based on a two-step procedure. First, a rational continuous-time stable model is built from the measured multiplexer's scattering parameters using the reader's preferred frequency domain system identification method for MIMO systems (a general method as [19] or a more dedicated one [23]). Structural properties make filters' transmission zeros play a key role in the algorithm: the values of the filters' reflection coefficients and a number of their derivatives evaluated at the (finite or infinite) transmission zeros can be decrypted from those of the multiplexer. This leads to a multipoint rational interpolation problem with derivative constraints, where the interpolation conditions are located at the filters' transmission zeros. Hence, the second step of 
the proposed strategy yields a rational representation of the scattering parameter matrices of the filters by solving this interpolation problem for each filter successively. Due to the inherent indetermination, filters are recovered up to a constant matrix (as shown in Sect. 5.4), which, in practice, corresponds to the resonator closest to the junction (see [27]).

Previous publications on this topic [20,26,27] address the same problem in a similar way, by regarding de-embedding as an interpolation problem, but develop different techniques. [20] presents a method based on a recursive Schur algorithm for the ideal case where filters are assumed lossless and measurements are exact. Subsequent papers [26,27] propose an alternative solution to the same Padé rational interpolation problem by determining the coefficients of two pairs of polynomials from an overdetermined linear system. The obtained polynomials yielded the rational scattering matrices of each filter and the approach was validated on real world examples (e.g., a manifold diplexer manufactured in one piece). In the present paper, instead, we propose a linear fractional representation of the solutions to the associated interpolation problem. This provides insight into the theoretic foundations of the method, a unified framework to compare the ideal and practical cases and last, but not least, allows for easy state-space computations.

The paper is organized as follows. Section 2 describes general concepts related to the problem we address. Section 3 shows that, due to structural properties, the de-embedding problem can be regarded as an interpolation problem with derivative constraints at the filters' transmission zeros. We show how to determine the transmission zeros and the interpolation values in Sect. 4, while Sect. 5 provides all solutions to this rational interpolation problem. Sect. 6 shows that the proposed algorithm applied to exact data obtained from lossless devices translates to Nevanlinna-Pick interpolation. Lastly, Sect. 7 validates the method on several numerical examples.

\section{Background}

This section starts by introducing notation. Lowercase boldface letters (e.g., v) denote column vectors, uppercase boldface letters (e.g., A) denote matrices, while non-boldface letters denote scalar quantities. If $\mathbf{M}$ is a complex matrix, $\mathbf{M}^{T}$ is its transpose and $\mathbf{M}^{*}$ is its complex conjugate transpose; $\mathbf{A} \geq \mathbf{B}$ (resp. $\mathbf{A}>\mathbf{B}$ ) means that the matrix $\mathbf{A}-\mathbf{B}$ is positive semidefinite (resp. definite). If $\mathbf{F}(s)$ is a matrix valued function, then $\mathbf{F}^{*}(s)=\mathbf{F}(-\bar{s})^{*}$ is the para-Hermitian conjugate of $\mathbf{F}(s)$, where $\bar{s}$ denotes the complex conjugate of $s$. Last, $\mathrm{i}$ denotes the unit imaginary number $\mathrm{i}=\sqrt{-1}$ and $\mathbb{C}^{+}$ denotes the open right-half of the complex plane.

\subsection{What is a filter?}

The term "filter" refers to a 2-port microwave device with a prescribed linear time invariant (LTI) response (Fig. 1 and 2 ). Scattering parameters ( $S$-parameters) relate the power

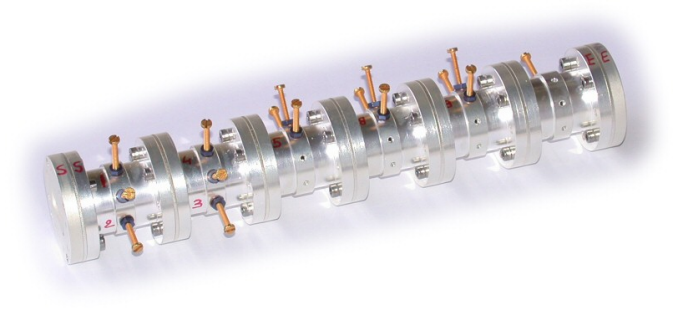

Fig. 1. A microwave filter

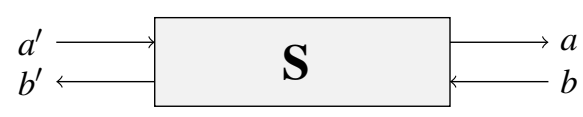

Fig. 2. Filtering device with incoming and outgoing power waves

of outgoing (reflected) waves to incoming (incident) waves. For a 2-port network (as in Fig. 2), we have that

$$
\left[\begin{array}{l}
a(\mathrm{i} \omega) \\
b^{\prime}(\mathrm{i} \omega)
\end{array}\right]=\mathbf{S}(\mathrm{i} \omega)\left[\begin{array}{l}
b(\mathrm{i} \omega) \\
a^{\prime}(\mathrm{i} \omega)
\end{array}\right],
$$

where $\omega=2 \pi f$, with $f$ being the excitation frequency, $a^{\prime}, b$, the incident waves and $a, b^{\prime}$, the reflected waves. Our notation differs from the standard of using $a, a^{\prime}$ for incoming and $b, b^{\prime}$ for outgoing waves. When connecting filters to a common junction and regarding the network as a multiplexer, the waves at the common ports are incoming for the junction (hence they could be denoted by $a$ ) and outgoing for the filter (hence denoted by $b$ ). To avoid double notations and keep the notation simple, we define the waves as in Fig. 2.

The scattering matrix is a proper rational matrix function and, for reciprocal filters, it is symmetric [3, Th. 2.8.1]:

$$
\mathbf{S}(s)=\frac{1}{q(s)}\left[\begin{array}{rr}
p_{1}(s) & t(s) \\
t(s) & p_{2}(s)
\end{array}\right]
$$

where $p_{1}(s), p_{2}(s), q(s)$ and $t(s)$ are polynomials. The order $n$ (or McMillan degree) of the filter is given by the degree of $q(s)$, provided that the condition $q$ divides $p_{1} p_{2}-t^{2}$ is satisfied [3]. The roots of $t(s)$ are referred to as the finite transmission zeros of the filter. There are $n$ transmission zeros, with the difference between $n$ and the degree of $t(s)$ yielding the number of transmission zeros at infinity. In practice, at least one transmission zero at infinity is assumed, so that $t(s)$ is of degree smaller or equal to $n-1$.

Transfer scattering or chain parameters (T-parameters) relate waves at one port to waves at the opposite port:

$$
\left[\begin{array}{l}
b^{\prime} \\
a^{\prime}
\end{array}\right]=\mathbf{T}\left[\begin{array}{l}
b \\
a
\end{array}\right] .
$$

T-parameters cannot be measured directly, unlike Sparameters, but they can be easily obtained from Sparameters (see Prop. 2.1). However, the representation in 
terms of T-parameters is especially useful when cascading devices, as the T-parameters of the interconnection are obtained by multiplying the T-parameters of the components.

Proposition 2.1 The filter's scattering and transfer scattering matrices defined by (1) and (3) are related by:

$$
\mathbf{T}(s)=\left[\begin{array}{cc}
S_{21}(s)-\frac{S_{11}(s) S_{22}(s)}{S_{12}(s)} & \frac{S_{22}(s)}{S_{12}(s)} \\
-\frac{S_{11}(s)}{S_{12}(s)} & \frac{1}{S_{12}(s)}
\end{array}\right]
$$

which is known as the Ginzburg transform. $\mathbf{T}(s)$ is defined when $S_{12}(s)$ is non-zero. The filter is reciprocal if and only if $\operatorname{det}(\mathbf{T}(s))=1$.

\subsection{Description of a multiplexer}

We consider a multiplexer composed of an $N+1$-port junction and $N$ filtering devices (Fig. 3 and 4).

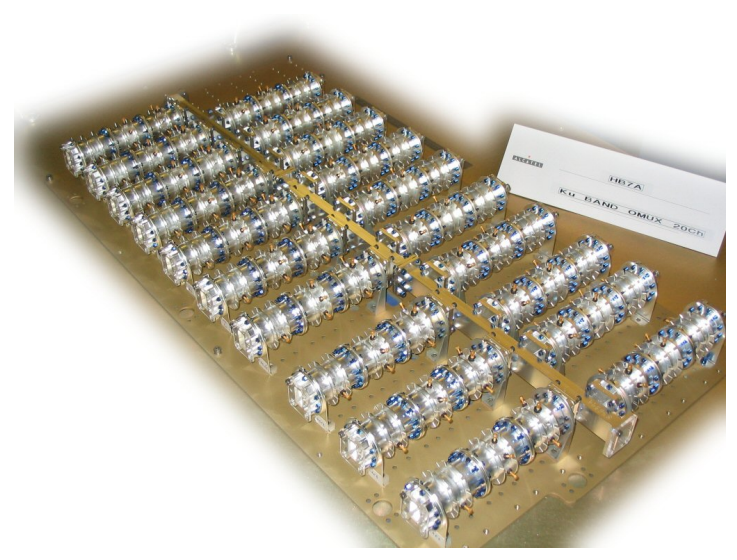

Fig. 3. A microwave multiplexer

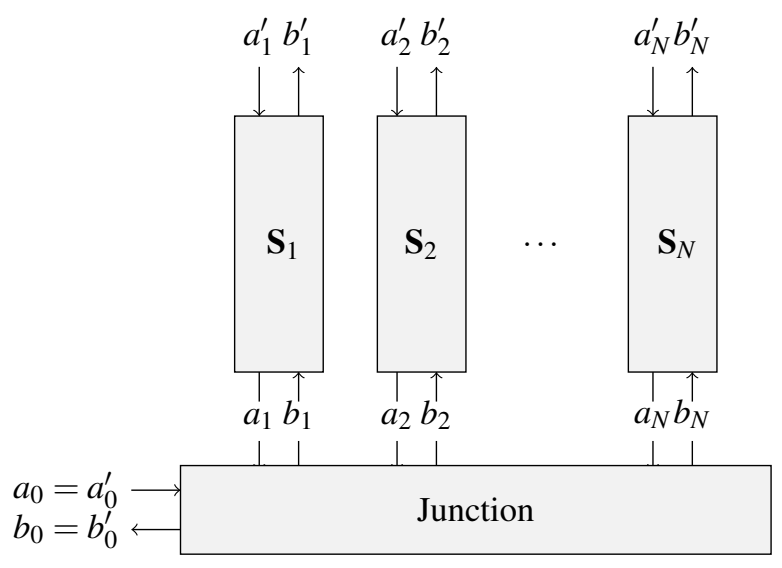

Fig. 4. Multiplexer

Let the waves $a_{k}, b_{k}, a_{k}^{\prime}$ and $b_{k}^{\prime}, k=1, \ldots, N$ be defined as in Fig. 4. We introduce vectors

$$
\begin{aligned}
\mathbf{a} & =\left[\begin{array}{llll}
a_{0} & a_{1} & \ldots & a_{N}
\end{array}\right]^{T}, \mathbf{b}=\left[\begin{array}{llll}
b_{0} & b_{1} & \ldots & b_{N}
\end{array}\right]^{T}, \\
\mathbf{a}^{\prime} & =\left[\begin{array}{llll}
a_{0}^{\prime} & a_{1}^{\prime} & \ldots & a_{N}^{\prime}
\end{array}\right]^{T}, \mathbf{b}^{\prime}=\left[\begin{array}{llll}
b_{0}^{\prime} & b_{1}^{\prime} & \ldots & b_{N}^{\prime}
\end{array}\right]^{T},
\end{aligned}
$$

so that

$$
\begin{aligned}
\mathbf{b} & =\widetilde{\boldsymbol{\Sigma}} \mathbf{a}, \\
\mathbf{b}^{\prime} & =\boldsymbol{\Sigma} \mathbf{a}^{\prime},
\end{aligned}
$$

where $\widetilde{\Sigma}$ and $\boldsymbol{\Sigma}$ denote the scattering matrices of the junction and of the multiplexer, respectively. The junction is assumed reciprocal. The scattering matrix $\mathbf{S}_{k}(s)$ and the transfer scattering matrix $\mathbf{T}_{k}(s)$ of filter number $k$, for $k=1, \ldots, N$, are

$$
\left[\begin{array}{c}
a_{k} \\
b_{k}^{\prime}
\end{array}\right]=\mathbf{S}_{k}\left[\begin{array}{l}
b_{k} \\
a_{k}^{\prime}
\end{array}\right], \quad\left[\begin{array}{l}
b_{k}^{\prime} \\
a_{k}^{\prime}
\end{array}\right]=\mathbf{T}_{k}\left[\begin{array}{l}
b_{k} \\
a_{k}
\end{array}\right] .
$$

This paper deals with (LTI, finite dimensional) passive networks, namely networks that do not generate energy. This translates to their rational scattering matrix $\Sigma(s)$ being Schur (or bounded real if it satisfies $\overline{\boldsymbol{\Sigma}(s)}=\boldsymbol{\Sigma}(\bar{s})$ ) [17, chap. 2]:

(1) $\boldsymbol{\Sigma}(s)$ is analytic for $s \in \mathbb{C}^{+}$,

(2) $\boldsymbol{\Sigma}(s)^{*} \boldsymbol{\Sigma}(s) \leq \mathbf{I}$ for $s \in \mathbb{C}^{+}$.

The second condition ensures that the system is stable (in the vicinity of a pole, a rational function cannot be bounded) and $\boldsymbol{\Sigma}(s)$ is also contractive on the imaginary axis [3, Th. 2.6.2]. Note that we allow for complex rational scattering matrices as they arise in the representation of narrow-band microwave devices using low-pass prototypes [25].

\subsection{Linear fractional representations}

Linear fractional transformations (LFT) are useful when describing interconnections between networks.

Let $\Theta(s)$ be a $2 p \times 2 p$ rational matrix function partitioned as

$$
\boldsymbol{\Theta}(s)=\left[\begin{array}{l|l}
\boldsymbol{\Theta}_{11}(s) & \boldsymbol{\Theta}_{12}(s) \\
\hline \boldsymbol{\Theta}_{21}(s) & \boldsymbol{\Theta}_{22}(s)
\end{array}\right]
$$

with blocks of size $p \times p$. The linear fractional transformation $\mathscr{T}_{\Theta}$ associated to $\Theta(s)$ takes any $p \times p$ matrix function $\mathbf{G}(s)$, for which $\boldsymbol{\Theta}_{21} \mathbf{G}+\boldsymbol{\Theta}_{22}$ is invertible, to the $p \times p$ matrix

$$
\mathscr{T}_{\boldsymbol{\Theta}}(\mathbf{G})=\left(\boldsymbol{\Theta}_{11} \mathbf{G}+\boldsymbol{\Theta}_{12}\right)\left(\boldsymbol{\Theta}_{21} \mathbf{G}+\boldsymbol{\Theta}_{22}\right)^{-1} \text {. }
$$

Linear fractional transformations satisfy the following properties: $\mathscr{T}_{\Theta}^{-1}(\mathbf{G})=\mathscr{T}_{\Theta^{-1}}(\mathbf{G})$ and $\mathscr{T}_{\Theta}\left(\mathscr{T}_{\boldsymbol{\Phi}}(\mathbf{G})\right)=\mathscr{T}_{\Theta \Phi}(\mathbf{G})$, where $\Theta \Phi$ denotes the product of the two matrix functions, with the composition of mappings $\mathscr{T}_{\Theta} \circ \mathscr{T}_{\boldsymbol{\Phi}}$ agreeing with $\mathscr{T}_{\Theta \Phi}$ on their joint domain of definition. 
Remark 2.1 [24, Th. 4] If the LFT $\Theta(s)$ satisfies

$$
\boldsymbol{\Theta}(s)^{-1}=\left[\begin{array}{rr}
\mathbf{0} & \mathbf{I} \\
-\mathbf{I} & \mathbf{0}
\end{array}\right] \boldsymbol{\Theta}(s)^{T}\left[\begin{array}{rr}
\mathbf{0} & -\mathbf{I} \\
\mathbf{I} & \mathbf{0}
\end{array}\right],
$$

then $\mathbf{F}=\mathscr{T}_{\Theta}(\mathbf{G})$ is symmetric if $\mathbf{G}$ is symmetric.

The Ginzburg transform relating scattering and transfer scattering matrix representations of a filter can be expressed in terms of a linear fractional transformation (see $[11,18]$ ):

$$
\mathbf{T}(s)=\mathscr{T}_{\mathbf{U}}(\mathbf{S}(s)) \text { with } \mathbf{U}=\left[\begin{array}{ll|ll}
0 & 1 & 0 & 0 \\
0 & 0 & 0 & 1 \\
\hline 0 & 0 & 1 & 0 \\
1 & 0 & 0 & 0
\end{array}\right] .
$$

Moreover, $\mathbf{S}(s)$ is Schur (passive filter) if and only if $\mathbf{T}(s)$ is $\mathbf{J}$-contractive [11, Th.1.1]:

$$
\mathbf{T}(s)^{*} \mathbf{J T}(s) \leq \mathbf{J}, s \in \mathbb{C}^{+}, \mathbf{J}=\operatorname{diag}(1,-1) .
$$

Remark 2.2 Note that $S_{22}(s)=\mathscr{T}_{\mathbf{T}(s)}(0)$. This corresponds to eliminating the reflected wave at the terminated port, thus closing this port on a matched termination.

Proposition 2.2 [18, Sect. 4.6] The multiplexer's scattering matrix $\boldsymbol{\Sigma}(s)$ can be computed as a linear fractional transformation of the junction's scattering matrix $\widetilde{\Sigma}$

$$
\boldsymbol{\Sigma}=\mathscr{T}_{\mathbf{T}}(\widetilde{\boldsymbol{\Sigma}})=\left(\mathbf{T}_{11} \widetilde{\boldsymbol{\Sigma}}+\mathbf{T}_{12}\right)\left(\mathbf{T}_{21} \widetilde{\boldsymbol{\Sigma}}+\mathbf{T}_{22}\right)^{-1}
$$

or alternatively as [2, Sect. 4]

$$
\boldsymbol{\Sigma}=\mathbf{S}_{22}+\mathbf{S}_{21} \widetilde{\boldsymbol{\Sigma}}\left(\mathbf{I}_{N+1}-\mathbf{S}_{11} \widetilde{\boldsymbol{\Sigma}}\right)^{-1} \mathbf{S}_{12}
$$

where $\mathbf{S}_{11}, \mathbf{S}_{12}, \mathbf{S}_{21}, \mathbf{S}_{22}$ and $\mathbf{T}_{11}, \mathbf{T}_{12}, \mathbf{T}_{21}, \mathbf{T}_{22}$ are defined as the following diagonal matrices

$$
\begin{aligned}
& \mathbf{S}_{i i}=\operatorname{diag}\left[\begin{array}{lllll}
0 & S_{1, i i} & S_{2, i i} & \ldots & S_{N, i i}
\end{array}\right], i=1,2, \\
& \mathbf{S}_{i j}=\operatorname{diag}\left[\begin{array}{lllll}
1 & S_{1, i j} & S_{2, i j} & \ldots & S_{N, i j}
\end{array}\right], i \neq j, i, j=1,2, \\
& \mathbf{T}_{i i}=\operatorname{diag}\left[\begin{array}{lllll}
1 & T_{1, i i} & T_{2, i i} & \ldots & T_{N, i i}
\end{array}\right], i=1,2, \\
& \mathbf{T}_{i j}=\operatorname{diag}\left[\begin{array}{lllll}
0 & T_{1, i j} & T_{2, i j} & \ldots & T_{N, i j}
\end{array}\right], i \neq j, i, j=1,2 .
\end{aligned}
$$

Note that the junction and the filters being passive, the inverse in (14) exists for $s \in \mathbb{C}^{+}$[11, Chap.3].

\section{Stating de-embedding as an interpolation problem}

The de-embedding problem can be formulated as follows:
Given $N_{s}$ frequency domain measurements of the multiport $S$ parameter matrix of a multiplexer, where $\Sigma_{i} \in \mathbb{C}^{(N+1) \times(N+1)}$ are the measurements provided at excitation frequency $f_{i}$, for $i=1, \ldots, N_{s}$, find a rational model for each of the $N$ passive reciprocal filters of known order composing the multiplexer. We assume no knowledge of the junction $\widetilde{\Sigma}$, except that it is passive and reciprocal.

We shall see that, due to structural properties, in particular (14), de-embedding can be regarded as a rational interpolation problem with interpolation conditions located at the transmission zeros of the filters. We first give the intuition behind this fact before formalizing it in Prop. 3.1.

Regarding the multiplexer as filter $k$ terminated by a load composed of the junction and the rest of the filters (Fig. $5)$, the load is not visible at frequency $\sigma$ coinciding with a transmission zero of the filter, as the incoming waves are reflected back to their respective output ports.

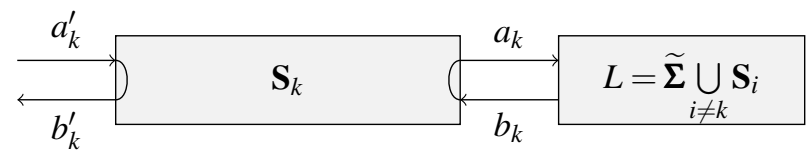

Fig. 5. Everything cascaded after the filter is not visible at the transmission zero

From Fig. 5, we conclude that

$$
b_{k}^{\prime}(\sigma)=\Sigma_{k+1, k+1}(\sigma) a_{k}^{\prime}(\sigma),
$$

where $\Sigma_{k+1, k+1}$ is the $k+1$ diagonal entry of the multiplexer's scattering matrix. On the other hand, rewriting (7) at $\sigma$ using that $S_{k, 12}(\sigma)=0$, leads to $b_{k}^{\prime}(\sigma)=S_{k, 22}(\sigma) a_{k}^{\prime}(\sigma)$. This, together with (19), yields

$$
S_{k, 22}(\sigma)=\Sigma_{k+1, k+1}(\sigma) .
$$

For a transmission zero $\sigma$ with multiplicity 1 , interpolation occurs for the value, as in (20), but also for the first derivative. In general, for $\sigma$ with multiplicity $m$, interpolation holds for the first $2 m-1$ derivatives of $S_{22}$ evaluated at $\sigma$. Zeros with higher multiplicity typically occur at infinity.

Proposition 3.1 Let $\sigma$ be a (possibly infinite, in which case evaluations in (21) are replaced by limits going to infinity) transmission zero of multiplicity $m$ for filter number $k, k \in$ $\{1, \ldots, N\}$. We assume that $\mathbf{I}-\mathbf{S}_{11}(\sigma) \widetilde{\boldsymbol{\Sigma}}(\sigma)$ is non singular. The following interpolation conditions hold:

$\left\{\begin{array}{l}\boldsymbol{\Sigma}^{(h)}(\sigma) \mathbf{e}_{k+1}=S_{k, 22}^{(h)}(\sigma) \mathbf{e}_{k+1}, h=0, \ldots, m-1, \\ \mathbf{e}_{k+1}^{T} \boldsymbol{\Sigma}^{(h)}(\sigma)=\mathbf{e}_{k+1}^{T} S_{k, 22}^{(h)}(\sigma), h=0, \ldots, m-1, \\ \sum_{k+1, k+1}^{(h)}(\sigma)=S_{k, 22}^{(h)}(\sigma), h=m, \ldots, 2 m-1,\end{array}\right.$

with $(\cdot)^{(h)}$ denoting the derivative of order $h$ and $\mathbf{e}_{k+1}$, the $k+1$ unit vector.

Conversely, if interpolation conditions in (21) hold at some 
point $\sigma$, then $\sigma$ is a transmission zero of filter $k$, unless $\sigma$ is a zero of the junction or a common zero to all other filters.

Proof. See Appendix A.

Remark 3.1 This result assumes that the inverse of $\mathbf{I}-$ $\mathbf{S}_{11}(\sigma) \widetilde{\boldsymbol{\Sigma}}(\sigma)$ exists. This condition is not always satisfied, as a transmission zero can be hidden in the connection (by simplification with a pole). The proof of Proposition 3.1 is purely algebraic and only makes use of the reciprocity of the filters, without requiring their passivity. However, if we assume passivity and also that the submatrix of $\tilde{\boldsymbol{\Sigma}}(s)$ built from the last $N$ rows and columns is strictly contractive, then, for any transmission zero $\sigma$ in the closed right halfplane, $\mathbf{I}_{N+1}-\mathbf{S}_{11}(\sigma) \widetilde{\boldsymbol{\Sigma}}(\sigma)$ is invertible [2].

Our paper relies on the interpolation conditions in (21) to identify the unknown filters. They relate information about the $(2,2)$ entry of the filter's S-parameters to quantities not readily available, but easily computable. The left-hand side can be determined from a continuous-time MIMO stable rational model $\boldsymbol{\Sigma}(s)$ built from the given measurements of the multiplexer. First, we find the transmission zeros of each filter $k$ as the common zeros of the non-diagonal entries of column and row $k+1$ of $\boldsymbol{\Sigma}(s)$. Therefore, previous knowledge on the location of the transmission zeros is not required. Second, (21) shows that de-embedding is essentially a rational interpolation problem for $S_{k, 22}(s)$. Denoting $S_{k, 22}(s)$ by $F(s)$, it satisfies the following interpolation problem.

Problem 3.1 Consider $n_{d}$ distinct finite interpolation points $\sigma_{1}, \ldots, \sigma_{n_{d}}$ with multiplicities $m_{1}, \ldots, m_{n_{d}}$, respectively, such that $m_{1}+\ldots+m_{n_{d}}=n_{f}$, together with the corresponding interpolation values $v_{1,0}, \ldots v_{1,2 m_{1}-1}, \ldots, v_{n_{d}, 0}, \ldots, v_{n_{d}, 2 m_{n_{d}}-1}$. In the double index notation, the first is the index of the transmission zero $\sigma$, while the second refers to the order of the derivative evaluated at that particular $\sigma$. Consider also the interpolation values at infinity: $v_{0,0}, \ldots, v_{0,2 n_{\infty}-1}$, with $n_{\infty}=n-n_{f}$. All values $v_{i, j}$ are finite.

Determine the rational function $F(s)$ of degree $n$ such that it satisfies the following $2 n$ interpolation conditions:

$$
\begin{aligned}
& F(s)^{(j)}\left(\sigma_{i}\right)=v_{i, j}, \forall i=1, \ldots, n_{d} \text { and } \forall j=0, \ldots, 2 m_{i}-1, \\
& \lim _{s \rightarrow \infty} F(s)^{(j)}(s)=v_{0, j}, \forall j=0, \ldots, 2 n_{\infty}-1 .
\end{aligned}
$$

The interpolation values mentioned above are found by evaluating the $k+1$ diagonal entry of the continuous-time MIMO model $\boldsymbol{\Sigma}(s)$, together with $2 m-1$ derivatives, at the transmission zeros of each filter. The rational interpolation problem stated above is a Padé multipoint problem [10]. A rational function of degree $n$ is determined by $2 n+1$ coefficients, while Problem 3.1 only defines $2 n$ conditions, hence it provides a solution to the de-embedding problem up to an inherent slight indetermination, as shown in Sect. 5.4.

The proposed de-embedding approach is summarized in the following steps:
(1) Compute a stable rational model of the S-parameter matrix of the multiplexer from the given measurements.

(2) For each filter

(a) Find all transmission zeros and their multiplicity (Sect. 4.1)

(b) Compute the interpolation values (Sect. 4.2).

(c) Solve the interpolation problem to obtain a rational representation of the S-matrix (Sect. 5).

\section{Determining the constraints in the rational interpo- lation problem}

The proposed approach solves the rational interpolation Problem 3.1 for the $S_{22}$ entry of each filter $k, k=1, \ldots, N$. The transmission zeros $\sigma$ and the values $v$ are not readily available, but can be obtained by following Proposition 3.1.

\subsection{Determining the transmission zeros}

We emphasize that the filters' transmission zeros are not given, rather they are computed. For each filter $k$, (21) shows that any finite transmission zero $\sigma$ is the common zero of all non-diagonal entries of the $(k+1)^{s t}$ row and column of $\boldsymbol{\Sigma}(s)$. After adding the multiplicities of all finite transmission zeros, the multiplicity of the infinite zero is computed as $n_{\infty}=n-n_{f}$, where $n$ is the filter's order (a-priori known) and $n_{f}$ is the number of finite transmission zeros.

\subsection{Determining the interpolation values}

S-parameters accept a state-space representation [3]: the $(k+1)^{s t}$ diagonal entry of the S-matrix $\boldsymbol{\Sigma}(s)$ of the multiplexer can be written as $\Sigma(s)_{k+1, k+1}=\mathbf{c}^{T}(s \mathbf{I}-\mathbf{A})^{-1} \mathbf{b}+d$. For simplicity, $\Sigma(s)_{k+1, k+1}$ is denoted by $G(s)$ in the following.

As most practical filters have at least one infinite transmission zero, the variable change $s \mapsto \frac{1}{s}$ moves all interpolation points to finite locations. For finite transmission zeros, the interpolation points are now $\widetilde{\sigma}_{i}=\frac{1}{\sigma_{i}}, i=1, \ldots, n_{d}$, while the infinite one is $\widetilde{\sigma}_{0}=0$. If a filter were to have transmission zeros at 0 , the problem could be solved in two stages: first, for transmission zeros at finite locations, and afterwards, for the transmission zero at infinity (by the change of variable).

Interpolation values after the change of variable are found as follows. The state-space representation is $\widetilde{G}(s)=G\left(\frac{1}{s}\right)$, with $\widetilde{G}(s)=\widetilde{\mathbf{c}}^{T}(s \mathbf{I}-\widetilde{\mathbf{A}})^{-1} \widetilde{\mathbf{b}}+\widetilde{d}$, where $\widetilde{\mathbf{A}}=\mathbf{A}^{-1}, \widetilde{\mathbf{b}}=\mathbf{A}^{-1} \mathbf{b}$, $\widetilde{\mathbf{c}}^{T}=-\mathbf{c}^{T} \mathbf{A}^{-1}$ and $\widetilde{d}=d-\mathbf{c}^{T} \mathbf{A}^{-1} \mathbf{b}$. The system $\widetilde{G}(s)$ is also proper, passive (thus stable) and of the same order as $G(s)$. The map $s \mapsto \frac{1}{s}$ is simply the low pass to high pass transformation. The interpolation values are found by evaluating $\widetilde{G}(s)$ and its first $2 m_{i}-1$ derivatives at $\widetilde{\sigma}_{i}$ :

$$
\widetilde{v}_{i, j}=\frac{1}{j !} \widetilde{G}^{(j)}\left(\widetilde{\sigma}_{i}\right)=(-1)^{j} \widetilde{\mathbf{c}}^{T}\left(\widetilde{\sigma}_{i} \mathbf{I}-\widetilde{\mathbf{A}}\right)^{-(j+1)} \widetilde{\mathbf{b}},
$$

is the $j^{t h}$ Taylor coefficient, where $j$ ! is the factorial. 


\section{Solving the rational interpolation problem}

This section constitutes the core of the proposed deembedding approach. After the change of variable $s \mapsto \frac{1}{s}$, the following problem is solved instead of Problem 3.1:

Problem 5.1 Given finite $\widetilde{\sigma}_{i}$, for $i=0, \ldots, n_{d}$, with multiplicities $m_{i}$ satisfying $\sum_{i=0}^{n_{d}} m_{i}=n$, find all rational proper functions $H(s)$ of order $n$ such that

$$
\frac{H^{(j)}\left(\widetilde{\sigma}_{i}\right)}{j !}=\widetilde{v}_{i, j}, \forall i=0, \ldots, n_{d} \text { and } \forall j=0, \ldots, 2 m_{i}-1 .
$$

All values $\widetilde{v}_{i, j}$ are finite. The quantity $H(s)$ stands for the unknown $S_{k, 22}\left(\frac{1}{s}\right)$.

The set of conditions in Problem 5.1 can be rewritten in contour integral form (see [6, Chap.16]). For a suitable choice of $n \times n$ matrices $\mathbf{M}$ (the spectrum of $\mathbf{M}$ being $\lambda(\mathbf{M})=$ $\left.\left\{\widetilde{\sigma}_{0}, \widetilde{\sigma}_{1}, \ldots, \widetilde{\sigma}_{n_{d}}\right\}\right)$ and $\mathbb{L}$, together with vectors $\mathbf{u}$ and $\mathbf{v}$, the interpolation conditions take the form

$$
\begin{aligned}
& \frac{1}{2 \pi \mathrm{i}} \int_{\Gamma}(s I-\mathbf{M})^{-1} \mathbf{u} H(s) d s=\mathbf{v}, \\
& \frac{1}{2 \pi \mathrm{i}} \int_{\Gamma}(s I-\mathbf{M})^{-1} \mathbf{u} H(s) \mathbf{u}^{T}\left(s I-\mathbf{M}^{T}\right)^{-1} d s=\mathbb{L},
\end{aligned}
$$

where $\Gamma$ is any suitable contour such that all $\tilde{\sigma}_{i}$ 's are inside $\Gamma$ and all poles of $H(s)$ are outside $\Gamma$. The matrix $\mathbb{L}$ can be viewed as a coupling matrix between left and right interpolation conditions.

In the following subsections, $\mathbf{M}, \mathbf{u}, \mathbf{v}$ and $\mathbb{L}$ are given for the case of all transmission zeros having single multiplicity, as well as for two transmission zeros with multiplicities $m_{1}$ and $m_{2}$. The general case can be extended from these two [1].

\subsection{Transmission zeros with single multiplicity}

Assuming that $\widetilde{\sigma}_{i} \neq \widetilde{\sigma}_{j}$, for $i \neq j$, define

$$
\begin{aligned}
& \mathbf{M}=\operatorname{diag}\left(\widetilde{\sigma}_{1}, \ldots, \widetilde{\sigma}_{n}\right) \in \mathbb{C}^{n \times n}, \\
& \mathbf{u}=\left[\begin{array}{lll}
1 & \ldots & 1
\end{array}\right]^{T} \in \mathbb{R}^{n \times 1}, \\
& \mathbf{v}=\left[\begin{array}{lll}
\widetilde{v}_{1,0} & \ldots & \widetilde{v}_{n, 0}
\end{array}\right]^{T} \in \mathbb{C}^{n \times 1}, \\
& \mathbb{L}_{i j}=\left\{\begin{array}{c}
\widetilde{v}_{i, 1}, \quad i=j \\
\widetilde{v}_{i, 0}-\widetilde{v}_{j, 0} \\
\frac{\widetilde{\sigma}_{i}-\widetilde{\sigma}_{j}}{\sigma^{\prime}}, i \neq j
\end{array}, i, j=1, \ldots, n .\right.
\end{aligned}
$$

5.2 Transmission zeros $\widetilde{\sigma}_{1}$ and $\widetilde{\sigma}_{2}$ with multiplicities $m_{1}$ and $m_{2}$, respectively

We define

$$
\mathbf{M}=\operatorname{blkdiag}\left(\mathbf{M}_{1}, \mathbf{M}_{2}\right) \in \mathbb{C}^{\left(m_{1}+m_{2}\right) \times\left(m_{1}+m_{2}\right)},
$$

where $\mathbf{M}_{1}^{T}=\mathbf{J}_{m_{1}}\left(\widetilde{\sigma}_{1}\right)$ is the Jordan block of size $m_{1}$ associated to $\widetilde{\sigma}_{1}$. Similarly for $\mathbf{M}_{2}^{T}=\mathbf{J}_{m_{2}}\left(\widetilde{\sigma}_{2}\right)$. We also define

$$
\begin{aligned}
& \mathbf{u}=\left[\begin{array}{l}
\mathbf{u}_{1} \\
\mathbf{u}_{2}
\end{array}\right]=\left[\begin{array}{llllll}
1 & \underbrace{0}_{m_{1}-1} \cdots & \cdots & \underbrace{0}_{m_{2}-1} & \cdots & 0
\end{array}\right]^{T} \in \mathbb{R}^{\left(m_{1}+m_{2}\right) \times 1} \text { and } \\
& \mathbf{v}=\left[\begin{array}{l}
\mathbf{v}_{1} \\
\mathbf{v}_{2}
\end{array}\right] \text { where } \mathbf{v}_{1}=\left[\begin{array}{lllll}
\widetilde{v}_{1,0} & \widetilde{v}_{1,1} & \ldots & \widetilde{v}_{1, j} & \widetilde{v}_{1, m_{1}-1}
\end{array}\right]^{T}
\end{aligned}
$$

and $\mathbf{v}_{2}$ is defined similarly. Last, we define the matrix

$$
\mathbb{L}=\left[\begin{array}{cc}
\mathbb{L}_{1} & \mathbb{L}_{12} \\
\mathbb{L}_{12}^{T} & \mathbb{L}_{2}
\end{array}\right] \text { where } \mathbb{L}_{1}=\left[\begin{array}{cccc}
\widetilde{v}_{1,1} & \widetilde{v}_{1,2} & \ldots & \widetilde{v}_{1, m_{1}} \\
\widetilde{v}_{1,2} & \widetilde{v}_{1,3} & \ldots & \widetilde{v}_{1, m_{1}+1} \\
\vdots & \vdots & \ddots & \vdots \\
\widetilde{v}_{1, m_{1}} & \widetilde{v}_{1, m_{1}+1} & \ldots & \widetilde{v}_{1,2 m_{1}-1}
\end{array}\right]
$$

is a Hankel matrix and $\mathbb{L}_{2}$ is a similar Hankel matrix containing $\widetilde{v}_{2,1}$ up to $\widetilde{v}_{2,2 m_{2}-1}$. The matrix $\mathbb{L}_{12}$ is the unique solution to the Sylvester equation

$$
\mathbb{L}_{12} \mathbf{M}_{2}^{T}-\mathbf{M}_{1} \mathbb{L}_{12}=\mathbf{u}_{1} \mathbf{v}_{2}^{T}-\mathbf{v}_{1} \mathbf{u}_{2}^{T} .
$$

is the so-called Loewner matrix. In the general case of distinct interpolation points $\widetilde{\sigma}_{0}, \ldots, \widetilde{\sigma}_{n}$ with multiplicities $m_{0}, \ldots, m_{n}, \mathbb{L}$ has Hankel blocks of size $m_{i}$ on the diagonal, for $i=0, \ldots, n$ [4], and blocks of size $m_{i} \times m_{j}$ on the off-diagonal, given by the solutions to (25).

\subsection{Defining the generating system $\Theta$}

The matrix $\mathbb{L}$ represents a key tool for studying parametrization issues for rational interpolation problems. It is known that Problem 5.1 has a solution of degree $n$ if and only if $\mathbb{L}$ is invertible $[4,5]$. The following theorem provides this solution in terms of the linear fractional transformations introduced in Sect. 2.3.

Making use of the quantities introduced in Sect. 5.1 and 5.2, we define the $2 \times 2$ rational matrix function $\Theta(s)$ as

$$
\boldsymbol{\Theta}(s)=\mathbf{I}+\left[\begin{array}{c}
\mathbf{v}^{T} \\
\mathbf{u}^{T}
\end{array}\right]\left(s \mathbf{I}-\mathbf{M}^{T}\right)^{-1} \mathbb{L}^{-1}\left[\begin{array}{ll}
\mathbf{u} & -\mathbf{v}
\end{array}\right] .
$$

Theorem 5.1 Assume that $\mathbb{L}$ is invertible and let $\delta \in \mathbb{C}$ be a constant such that

$$
\mathbf{X}_{\delta}(s)=s \mathbb{L}-\mathbb{L} \mathbf{M}^{T}+(\mathbf{u} \delta-\mathbf{v}) \mathbf{u}^{T}
$$

is invertible for $s$ in $\lambda(\mathbf{M})$. Then $H(s)=\mathscr{T}_{\Theta}(\delta)$ is a solution to the interpolation problem 5.1. Moreover, $H(s)$ is a proper rational function of degree $n$ and none of its poles coincide with an interpolation point.

Conversely, let $H(s)$ be a proper rational function of degree $n$ with no poles coinciding with an interpolation point, satisfying the interpolation problem 5.1. Then $\mathbb{L}$ is invertible 
and $H(s)$ can be represented by a $L F T H(s)=\mathscr{T}_{\Theta}(\delta)$, for some constant $\delta \in \mathbb{C}$, such that $\mathbf{X}_{\delta}(s)$ is invertible at any interpolation point.

Proof. See Appendix B.

\subsection{Linking $\Theta(s)$ to the de-embedding problem}

According to Theorem 5.1, the generating system $\Theta(s)$ in (26) describes all solutions to the interpolation problem 5.1. Returning to Problem 3.1, all solutions are given by the function $F_{\delta}(s)=\mathscr{T}_{\Theta\left(\frac{1}{s}\right)}(\delta)$ in terms of the $\delta$ parameter. This can be interpreted as $F_{\delta}(s)$ being the reflection parameter observed from the input of a filter with transfer scattering matrix $\Theta\left(\frac{1}{s}\right)$ closed on a constant $\delta$. If $\delta$ is regarded as the input reflection of a constant reciprocal filter with transfer parameters $\mathbf{T}_{c}$ closed on a matched termination $\mathscr{T}_{\mathbf{T}_{c}}(0)$, then $F_{\delta}(s)=\mathscr{T}_{\boldsymbol{\Theta}\left(\frac{1}{s}\right)}\left(\mathscr{T}_{\mathbf{T}_{c}}(0)\right)$. By the composition property of LFTs, we have that $F_{\delta}(s)=\mathscr{T}_{\boldsymbol{\Theta}\left(\frac{1}{s}\right) \mathbf{T}_{c}}(0)$. Defining $\mathbf{T}(s)=\boldsymbol{\Theta}\left(\frac{1}{s}\right) \mathbf{T}_{c}$ as the cascade of the two, in view of Remark 2.2, this is the transfer scattering matrix of a filter

- which is reciprocal (symmetric), so $\operatorname{det}(\mathbf{T}(s))=1$,

- which has transmission zeros given by $\sigma_{i}$,

- for which $S_{22}(s)=F_{\delta}(s)$ satisfies the interpolation conditions in Problem 3.1,

- which is of degree $n$.

We have shown that the filter, described by its transfer scattering matrix $\mathbf{T}(s)$, is determined by $\boldsymbol{\Theta}\left(\frac{1}{s}\right)$, the solution to the interpolation problem 3.1, up to a constant transfer matrix $\mathbf{T}_{c}$, or equivalently, up to a constant symmetric matrix $\mathbf{S}_{c}=\mathscr{T}_{\mathbf{U}^{-1}}\left(\mathbf{T}_{c}\right)$ depending on three free parameters

$$
\mathbf{S}_{c}=\left[\begin{array}{ll}
\alpha & \beta \\
\beta & \delta
\end{array}\right]
$$

Let

$$
\boldsymbol{\Theta}\left(\frac{1}{s}\right)=\frac{\left[\begin{array}{ll}
-q_{1}(s) & p_{2}(s) \\
-p_{1}(s) & q_{2}(s)
\end{array}\right]}{t(s)},
$$

with $p_{1} p_{2}-q_{1} q_{2}=t^{2}$, be a polynomial representation of the transfer scattering matrix. This matrix can also be built from two independent solutions to the Padé rational interpolation problem 3.1, namely $\frac{p_{2}}{q_{2}}$ and $\frac{q_{1}}{p_{1}}$ [1], as was done in [26,27]. Note that $\frac{p_{2}}{q_{2}}$ is obtained as $\mathscr{T}_{\Theta\left(\frac{1}{s}\right)}(0)$ in the parametrization (9), while $\frac{q_{1}}{p_{1}}$ is obtained as $\mathscr{T}_{\boldsymbol{\Theta}\left(\frac{1}{s}\right)}(\infty)$ from (9). The role of the parameters $\alpha, \beta, \delta$ is clear when computing the scattering matrix $\mathbf{S}(s)$ associated with $\Theta\left(\frac{1}{s}\right) \mathbf{T}_{c}$ :

$$
\mathbf{S}(s)=\frac{\left[\begin{array}{cc}
\left(\beta^{2}-\alpha \delta\right) p_{1}(s)+\alpha q_{2}(s) & \beta t(s) \\
\beta t(s) & p_{2}(s)-\delta q_{1}(s)
\end{array}\right]}{q_{2}(s)-\delta p_{1}(s)}:
$$

$\beta$ determines the leading coefficient of the transmission polynomial; $\delta$ and $\alpha$ select a new couple of solutions to the Padé interpolation problem 3.1. This establishes the connection with Proposition 2.2 in [27].

The result of the proposed procedure is depicted in Fig. 6 . The transfer matrices of filters $k=1,2, \ldots, N$ are recovered as the $\mathbf{T}_{k}(s)$ matrix functions. Without knowing the junction, this de-embedding problem is underdetermined, hence the filters are recovered up to the factors $\mathbf{T}_{c k}$.

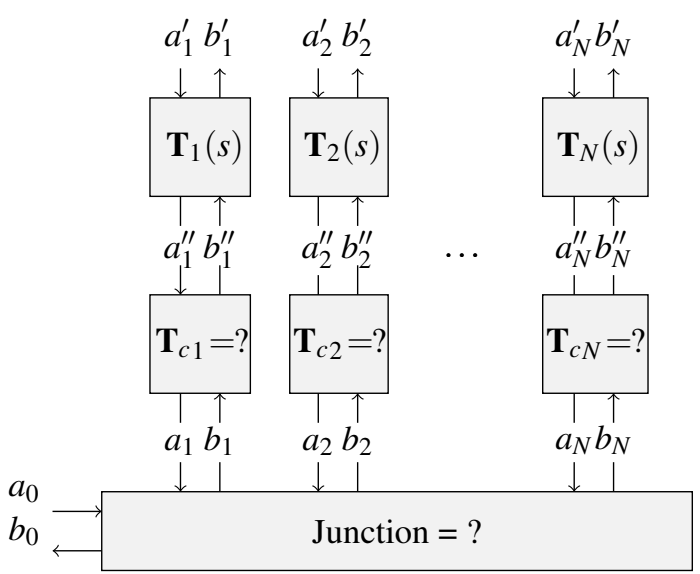

Fig. 6. Decomposition of the multiplexer in terms of recoverable and nonrecoverable quantities

\subsection{What about stability and passivity?}

Our approach preserves reciprocity for the recovered filters as this results from the interpolation conditions (Sect. 5.4). Filters are identified up to the resonator closest to the junction [27, Proposition 2.3], which cannot be found due to the inherent indetermination of the problem. All electrical parameters, except the offset of the last cavity and the output coupling, are recovered, thus allowing engineers to fine tune the screws to match the desired specifications. This good agreement also relies on the quality of the approximation step to provide reliable interpolation data.

However, our method guarantees neither stability, nor passivity. On the other hand, we describe all rational solutions of prescribed degree to our interpolation problem in Th. 5.1 so, if a passive solution does exist, it should be of this form. Naturally, it would be desirable to have conditions on the interpolation data which ensure that a passive solution does exist and to characterize these passive solutions among rational ones. Unfortunately, this is a hard problem, for which we do not have an answer yet.

Problem 5.1 can thus be viewed as a Nevanlinna-Pick type problem with $2 n$ interpolation conditions. While necessary and sufficient conditions for the existence of a solution (Pick's theorem) are known for a long time, the question of the degree is still relevant. A beautiful theory developed in [8] shows that all solutions of degree at most $2 n$ can be 
parametrized by spectral zeros. These solutions have generic degree $2 n$ and the corresponding filter is lossless with $n$ additional transmission zeros, the mirror images of the true ones. However, this is not what we want, as we are interested in minimum degree interpolants and this degree should be $n$. Unfortunately the issue of minimal degree interpolants is a hard problem, mentioned many times in the literature $[8,13,28]$. To the best of our knowledge, this is still an unsolved problem. For the moment, we are only able to ensure passivity of the filter in the ideal case of perfect data coming from a lossless filter, as shown in the following section.

\section{Ideal case: lossless data}

By ideal case, we refer to the case were filters are perfectly lossless and data are error-free, while the junction is still assumed to be passive. This section shows that, when our procedure is applied on lossless data, rational interpolation produces a lossless filter (still non-uniquely determined).

\subsection{Lossless and lossy passive devices}

A lossless or ideal device (be it a filter or a multiplexer) does not dissipate power, so incident and reflected powers are equal. This implies that the S-parameter matrix is unitary on the imaginary axis: $\boldsymbol{\Sigma}(\mathrm{i} \omega)^{*} \boldsymbol{\Sigma}(\mathrm{i} \omega)=\mathbf{I}$, for $\omega \in \mathbb{R}[9]$. A lossy or non ideal device dissipates power, so the incident power is greater than the reflected power. The property equivalent to losslessness for T-parameters is J-losslessness [18]: $\mathbf{T}(\mathrm{i} \omega)^{*} \mathbf{J T}(\mathrm{i} \omega)=\mathbf{J}$, for $\omega \in \mathbb{R}$, and $\mathbf{T}(s)^{*} \mathbf{J T}(s)<\mathbf{J}$, for $s \in \mathbb{C}^{+}$, where $\mathbf{J}=\operatorname{blkdiag}(\mathbf{I},-\mathbf{I})$.

The conditions for lossless (ideal) filters represented as in (2) translate to the polynomials $p_{1}, p_{2}$ and $t$ satisfying

$$
p_{2}(s)=p(s), \quad p_{1}(s)=\varepsilon p^{*}(s) \text { and } t(s)=-\varepsilon t^{*}(s)
$$

for $\varepsilon \in \mathbb{C},|\varepsilon|=1$. These conditions are known as the Belevitch representation [7] for ideal filters, yielding

$$
p(s) p^{*}(s)+t(s) t^{*}(s)=q(s) q^{*}(s)
$$

also known as the Feldtkeller equation when restricted to the imaginary axis:

$$
|p(\mathrm{i} \omega)|^{2}+|t(\mathrm{i} \omega)|^{2}=|q(\mathrm{i} \omega)|^{2} .
$$

Remark 6.1 [6,15] If $\boldsymbol{\Theta}(s)$ is $\mathbf{J}$-lossless, then $\mathbf{F}(s)=$ $\mathscr{T}_{\Theta(s)}(\mathbf{G}(s))$ is lossless (resp. Schur) if $\mathbf{G}(s)$ is a lossless (resp. Schur) matrix function.

6.2 Proposed de-embedding approach on noise-free measurements obtained in the ideal case

The ideal case corresponds to a multiplexer composed of lossless filters cascaded to a passive junction. For noise-free data, we show that the T-parameters of each filter obtained by solving the de-embedding problem via the proposed rational interpolation approach are $\mathbf{J}$-lossless and, consequently, the filter is lossless.

Proposition 6.1 Assume that data in Problem 5.1 are noisefree and obtained from a lossless and reciprocal filter with scattering matrix $\mathbf{S}(s)$. Let $\sigma$ be an interpolation point of multiplicity $m$, and denote the corresponding interpolation values $\frac{1}{k !}\left(\frac{p}{q}\right)^{(k)}(\sigma)$ by $v_{k}, k=0, \ldots, 2 m-1$. If $\sigma$ is not on the imaginary axis, then $\sigma^{\prime}=-\bar{\sigma}$ is also an interpolation point with the same multiplicity. Moreover, the interpolation values $\frac{1}{k !}\left(\frac{p}{q}\right)^{(k)}\left(\sigma^{\prime}\right)=\mu_{k}, k=0, \ldots, 2 m-1$, satisfy
$\left\{\begin{array}{l}\bar{v}_{0} \mu_{0}=1 \\ \sum_{h=0}^{k} \bar{v}_{k-h} \mu_{h}(-1)^{k-h}=0, \quad k=1, \ldots, 2 m-1 .\end{array}\right.$

If $\sigma$ is on the axis, then $\left|v_{0}\right|=1$ and (31) is satisfied with $\mu_{k}=v_{k}$ for $k=1, \ldots, 2 m-1$.

Proof. The transmission polynomial being auto-reciprocal $\left.\overline{\left(t^{*}(s)\right.}=-\varepsilon t(s)\right)$ implies that its zeros are either on the imaginary axis or in pairs which are symmetric with respect to the imaginary axis. Eq. (29) evaluated at $s=\sigma$ yields $v_{0} \overline{\mu_{0}}=1$. The $k^{\text {th }}$ derivative of (29) computed via the Leibniz rule is

$$
\sum_{h=0}^{k}\left(\begin{array}{l}
k \\
h
\end{array}\right)\left(\left(\frac{p}{q}\right)^{(h)}\left(\frac{p^{*}}{q^{*}}\right)^{(k-h)}+\left(\frac{t}{q}\right)^{(h)}\left(\frac{t^{*}}{q^{*}}\right)^{(k-h)}\right)=0,
$$

for $k \geq 1$, which gives the second relation in Eq. (31) when evaluated at $s=\sigma$. Transmission zeros on the imaginary axis satisfy (30) and its higher order derivatives, which immediately yields that $\mu_{k}=v_{k}$, for $k=1, \ldots, 2 m-1$.

Proposition $6.2 \Theta(s)$ in (26) associated with exact interpolation data obtained from an ideal multiplexer is J-lossless.

Proof. Let $\sigma$ be an interpolation point with multiplicity $m \geq$ 1. We denote by $\mathbf{M}_{\sigma}, \mathbf{u}_{\sigma}$ and $\mathbf{v}_{\sigma}$, the blocks of $\mathbf{M}, \mathbf{u}$ and $\mathbf{v}$ corresponding to $\sigma$, and similarly for $\sigma^{\prime}$. These are $\mathbf{v}_{\sigma}^{T}=$ $\left[\begin{array}{llll}v_{0} & v_{1} & \ldots & v_{m-1}\end{array}\right], \mathbf{v}_{\sigma^{\prime}}^{T}=\left[\begin{array}{llll}\mu_{0} & \mu_{1} & \ldots & \mu_{m-1}\end{array}\right]$, while $\mathbf{u}_{\sigma}^{T}=$ $\mathbf{u}_{\sigma^{\prime}}^{T}=\left[\begin{array}{llll}1 & 0 & \ldots & 0\end{array}\right]$.

Eq. (31) can be rewritten in a $m \times m$ matrix equation as

$$
\underbrace{\left[\begin{array}{ccccc}
\bar{v}_{0} & 0 & & \ldots & 0 \\
\bar{v}_{1} & \bar{v}_{0} & 0 & \ldots & 0 \\
\bar{v}_{2} & \bar{v}_{1} & \bar{v}_{0} & \ldots & 0 \\
\vdots & \vdots & \vdots & \ddots & \vdots
\end{array}\right]}_{\mathbf{T}_{\sigma}}\left[\begin{array}{lllll}
\mu_{0} & 0 & & \ldots & 0 \\
\mu_{1} & \mu_{0} & 0 & \ldots & 0 \\
\mu_{2} & \mu_{1} & \mu_{0} & \ldots & 0 \\
\vdots & \vdots & \vdots & \ddots & \vdots \\
& \ldots & \mu_{2} & \mu_{1} & \mu_{0}
\end{array}\right] \mathbf{D}=\mathbf{I},
$$

where $\mathbf{D}=\operatorname{diag}\left(1,-1, \ldots,(-1)^{m-1}\right)$. This shows that data at $\sigma$ and $\sigma^{\prime}$ are connected by 


$$
\mathbf{T}_{\sigma} \mathbf{v}_{\sigma^{\prime}}=\overline{\mathbf{u}}_{\sigma}, \quad \mathbf{T}_{\sigma} \mathbf{u}_{\sigma^{\prime}}=\overline{\mathbf{v}}_{\sigma}, \quad \mathbf{T}_{\sigma} \mathbf{M}_{\sigma^{\prime}} \mathbf{T}_{\sigma}^{-1}=-\overline{\mathbf{M}}_{\sigma}
$$

Collecting all $\mathbf{T}_{\sigma}$ corresponding to $\sigma \in \mathbb{C}^{+}$according to their order in $\mathbf{M}_{+}$into a block diagonal matrix $\mathbf{T}$, we obtain

$$
\mathbf{T} \mathbf{v}_{-}=\overline{\mathbf{u}}_{+}, \quad \mathbf{T} \mathbf{u}_{-}=\overline{\mathbf{v}}_{+}, \quad \mathbf{T} \mathbf{M}_{-} \mathbf{T}^{-1}=-\overline{\mathbf{M}}_{+} .
$$

For interpolation points on the imaginary axis, the $\mu_{i}$ 's and $v_{i}$ 's coincide. This allows to built the matrix $\mathbf{T}_{0}$ such that

$$
\mathbf{T}_{0} \mathbf{v}_{0}=\overline{\mathbf{u}}_{0}, \quad \mathbf{T}_{0} \mathbf{u}_{0}=\overline{\mathbf{v}}_{0}, \quad \mathbf{T}_{0} \mathbf{M}_{0} \mathbf{T}_{0}^{-1}=-\overline{\mathbf{M}}_{0} .
$$

We partition matrix $\mathbf{M}$ and vectors $\mathbf{u}$ and $\mathbf{v}$ in Sect. 5.1 and 5.2 according to the location of the interpolation points

$$
\mathbf{M}=\operatorname{blkdiag}\left(\mathbf{M}_{+}, \mathbf{M}_{0}, \mathbf{M}_{-}\right), \mathbf{u}=\left[\begin{array}{l}
\mathbf{u}_{+} \\
\mathbf{u}_{0} \\
\mathbf{u}_{-}
\end{array}\right], \mathbf{v}=\left[\begin{array}{c}
\mathbf{v}_{+} \\
\mathbf{v}_{0} \\
\mathbf{v}_{-}
\end{array}\right],
$$

where $\mathbf{M}_{+}^{T}, \mathbf{M}_{0}^{T}, \mathbf{M}_{-}^{T}$ contain all Jordan blocks associated to points in the right half-plane $\left(\mathbb{C}^{+}\right)$, on the imaginary axis, and in the left half-plane $\left(\mathbb{C}^{-}\right)$, respectively. Substituting (36) into (26) and using (34)-(35) yields

$\boldsymbol{\Theta}(s)=\mathbf{I}+\underbrace{\left[\begin{array}{lll}\mathbf{v}_{+}^{T} & \mathbf{v}_{0}^{T} & -\mathbf{u}_{+}^{*} \\ \mathbf{u}_{+}^{T} & \mathbf{u}_{0}^{T} & -\mathbf{v}_{+}^{*}\end{array}\right]}_{\mathbf{C}_{\boldsymbol{\Theta}}}(\underbrace{\left[\begin{array}{lll}\mathbf{M}_{+}^{T} & & \\ & \mathbf{M}_{0}^{T} & \\ & & \\ & & -\mathbf{M}_{+}^{*}\end{array}\right]}_{\mathbf{A}_{\boldsymbol{\Theta}}})^{-1} \widehat{\mathbb{L}}^{-1} \underbrace{\left[\begin{array}{cc}\mathbf{u}_{+} & -\mathbf{v}_{+} \\ \mathbf{u}_{0} & -\mathbf{v}_{0} \\ -\overline{\mathbf{v}}_{+} & \overline{\mathbf{u}}_{+}\end{array}\right]}_{\mathbf{B}_{\boldsymbol{\Theta}}}$

where $\widehat{\mathbb{L}}=\operatorname{blkdiag}(\mathbf{I}, \mathbf{I},-\mathbf{T}) \mathbb{L} \operatorname{blkdiag}(\mathbf{I}, \mathbf{I},-\mathbf{T})^{T}$ is symmetric. Multiplying $\mathbf{u}_{0}$ and $\mathbf{v}_{0}$ by $-\mathbf{T}_{0}$ and swapping the first and last row blocks in $\mathbf{B}_{\Theta}$, we obtain

$$
\begin{aligned}
& \boldsymbol{\Theta}(s)=\mathbf{I}+\mathbf{C}_{\boldsymbol{\Theta}}\left(s \mathbf{I}-\mathbf{A}_{\boldsymbol{\Theta}}\right)^{-1} \mathbb{P}^{-1}\left(-\mathbf{C}_{\boldsymbol{\Theta}}^{*} \mathbf{J}\right), \quad \text { where } \\
& \mathbb{P}=\left[\begin{array}{ll}
{ }^{-} \mathbf{T}_{0} & \\
\mathbf{I} &
\end{array}\right] \mathbb{L}\left[\begin{array}{lll}
\mathbf{I} & & \\
& \mathbf{I} & \\
& & -\mathbf{T}^{T}
\end{array}\right] .
\end{aligned}
$$

Using (31), it can be checked that $\mathbb{P}$ is Hermitian. Moreover, from $\mathbb{L} \mathbf{M}^{T}-\mathbf{M} \mathbb{L}=\mathbf{u v} \mathbf{v}^{T}-\mathbf{v u} \mathbf{u}^{T}$, it is easily established that

$$
\mathbb{P} \mathbf{A}_{\Theta}+\mathbf{A}_{\Theta}^{*} \mathbb{P}=-\mathbf{C}_{\Theta}^{*} \mathbf{J} \mathbf{C}_{\Theta} \text {. }
$$

According to [6, Th. 6.1.1], $\boldsymbol{\Theta}(s)$ is $\mathbf{J}$-unitary on the imaginary axis. Moreover, since the data comes from an ideal filter (of degree $n$ ), we know that there exists a solution which is analytic in the closed right half-plane. Then, by Theorem 21.4.1 in [6], $\mathbb{P}$ is positive definite and thus $J$-lossless.

Remark 6.2 In view of [6, Th 21.4.1], $\Theta$ is the generating system associated to a generalized boundary NevanlinnaPick interpolation problem. In this problem, only interpolation data occurring at $\sigma \in \mathbb{C}^{+} \bigcup\{\mathrm{i} \mathbb{R}\}$ are considered (the conditions at $\sigma \in \mathbb{C}^{-}$being automatically built in). Note that, when no interpolation points are on the boundary, we get the so-called central or maximum entropy solution since spectral zeros (transmission zeros) coincide with interpolation points (see [8]).

This provides a lossless (and thus stable) solution to the de-embedding problem: as in section 5.4, the scattering matrix of the filter is obtained by applying the inverse of the Ginzburg transform defined in Proposition 2.1 to $\Theta(1 / s)$. As before, the solution is not unique, all lossless solutions being obtained by multiplication of $\boldsymbol{\Theta}(1 / s)$ with an arbitrary constant $\mathbf{T}_{c}$, which must be chosen $\mathbf{J}$-unitary: $\mathbf{T}_{c}^{*} \mathbf{J} \mathbf{T}_{c}=\mathbf{J}$.

\section{Numerical examples}

\subsection{Recovering an a-priori known filter}

The $4^{\text {th }}$ order ideal filter is given by the reflection polynomial

$$
p(s)=s^{4}-3 \mathrm{is}^{3}-3.3107 s^{2}+1.591 \mathrm{i} s+0.2808
$$

together with the transmission polynomial

$$
t(s)=0.018 \mathrm{i} s^{2}+0.027 s-0.0047 \mathrm{i}
$$

The denominator $q(s)$ is simply found as the stable solution of (29). There are two finite transmission zeros at $1.3 \mathrm{i}$ and $0.2 \mathrm{i}$ and one infinite with multiplicity 2 . Table 1 lists the interpolation values $\widetilde{v}_{0,0}, \widetilde{v}_{1,0}, \widetilde{v}_{2,0}, \widetilde{v}_{0,1}, \widetilde{v}_{1,1}, \widetilde{v}_{2,1}, \widetilde{v}_{0,2}, \widetilde{v}_{0,3}$.

\begin{tabular}{|c|c|c|c|c|}
\hline Tr. zero & $\begin{array}{c}\text { value } \\
\widetilde{v}_{i, 0}\end{array}$ & $\begin{array}{c}1^{s t} \text { deriv. } \\
\widetilde{v}_{i, 1}\end{array}$ & $\begin{array}{c}2^{\text {nd }} \text { deriv. } \\
\widetilde{v}_{i, 2}\end{array}$ & $\begin{array}{c}3^{r d} \text { deriv. } \\
\widetilde{v}_{i, 3}\end{array}$ \\
\hline$\sigma_{0}=\infty$ & 1 & -0.53 & $0.28-0.79 \mathrm{i}$ & $1.71+1.25 \mathrm{i}$ \\
\hline$\sigma_{1}=1.3 \mathrm{i}$ & $0.49+0.87 \mathrm{i}$ & $-1.97-3.5 \mathrm{i}$ & N/A & N/A \\
\hline$\sigma_{2}=0.2 \mathrm{i}$ & $0.49-0.87 \mathrm{i}$ & $-0.05+0.08 \mathrm{i}$ & N/A & N/A \\
\hline
\end{tabular}

Table 1

Interpolation conditions

Defining the quantities

$$
\begin{aligned}
& \mathbf{M}^{T}=\left[\begin{array}{cccc}
\frac{1}{1.3 \mathrm{i}} & & & \\
& \frac{1}{0.2 \mathrm{i}} & \\
& 0 & 1 \\
& &
\end{array}\right], \mathbf{u}=\left[\begin{array}{l}
1 \\
1 \\
1 \\
0
\end{array}\right], \mathbf{v}=\left[\begin{array}{c}
0.49+0.87 \mathrm{i} \\
0.49-0.87 \mathrm{i} \\
1 \\
-0.53
\end{array}\right], \quad(38) \\
& \mathbb{L}=\left[\begin{array}{cccc}
-1.97-3.5 \mathrm{i} & 0.41 & -1.13-0.66 \mathrm{i} & 0.86-0.79 \mathrm{i} \\
0.41 & -0.05+0.08 \mathrm{i} & 0.17-0.1 \mathrm{i} & 0.02+0.14 \mathrm{i} \\
-1.13-0.66 \mathrm{i} & 0.17-0.1 \mathrm{i} & -0.53 & 0.14-0.4 \mathrm{i} \\
0.86-0.79 \mathrm{i} & 0.02+0.14 \mathrm{i} & 0.14-0.4 \mathrm{i} & 0.26+0.21 \mathrm{i}
\end{array}\right]
\end{aligned}
$$


and using the $\Theta$ as in (26) with Laplace variable $s$ replaced by $\frac{1}{s}$, we recover the filter up to the constant J-unitary matrix

$$
\mathbf{T}_{c}=\left[\begin{array}{cc}
-40.41+44.49 \mathrm{i} & -60.09 \mathrm{i} \\
60.09 \mathrm{i} & -40.41-44.49 \mathrm{i}
\end{array}\right] .
$$

Therefore, the transfer scattering matrix of the filter $\mathbf{T}(s)$ can be expressed as the product $\boldsymbol{\Theta}(1 / s) \mathbf{T}_{c}$.

\subsection{Recovering a-priori known filters from a diplexer with constant junction}

Consider a diplexer with two filters and the constant junction

$$
\boldsymbol{\Sigma}_{0}=\left[\begin{array}{rrr}
-\frac{1}{3} & \frac{2}{3} & \frac{2}{3} \\
\frac{2}{3} & -\frac{1}{3} & \frac{2}{3} \\
\frac{2}{3} & \frac{2}{3} & -\frac{1}{3}
\end{array}\right]
$$

The first filter is the same filter used in Sect. 7.1, while the second one is given by the following polynomials

$$
\begin{aligned}
p(s) & =s^{4}+3 \mathrm{i} s^{3}-3.3107 s^{2}-1.591 \mathrm{i} s+0.2808, \\
t(s) & =0.018 \mathrm{i} s^{2}-0.027 s-0.0047 \mathrm{i},
\end{aligned}
$$

with transmission zeros located at $-1.3 \mathrm{i},-.2 \mathrm{i}$ and infinity (with multiplicity 2). Due to the special choice of the polynomials for filter 2 , its interpolation values are complex conjugates of those listed in Table 1 , so $\mathbf{M}, \mathbf{u}, \mathbf{v}$ and $\mathbb{L}$ are complex conjugates of those in (38). The unrecoverable Junitary constant factor is found to be

$$
\mathbf{T}_{c}=\left[\begin{array}{cc}
40.41+44.49 \mathrm{i} & -60.09 \mathrm{i} \\
60.09 \mathrm{i} & 40.41-44.49 \mathrm{i}
\end{array}\right] .
$$

\subsection{De-embedding filters from a triplexer given by its $S$ - parameter computed via full-wave simulations}

We present a numerical example provided by Thales obtained from a full-wave simulation. For validation purposes, the filters' S-parameter are also known.

This set contains S-parameter data of a triplexer for 1000 frequencies linearly distributed between 11.4 and $11.6 \mathrm{GHz}$. For convenience, frequencies are scaled so that they span the interval $[-1,1]$. For step (1) of the algorithm, we identify an order 16 model for the triplexer which matches the data with $10^{-7}$ accuracy using the approach presented in $[19,21]$.

For filter 1, the transmission zeros are identified as the common zeros of the $2^{\text {nd }}$ column of the multi-port model of the triplexer. They are found to be located at $-.004-$
$.559 \mathrm{i},-.004-.064 \mathrm{i}$ and infinity with multiplicity 2 . The finite transmission zeros, ideally purely imaginary, have nonnegligible real part, so the filter is slightly lossy. To compute the interpolation values at the transmission zeros, we evaluate the SISO system given by the diagonal entry $(2,2)$ of the 4-port MIMO model of the triplexer. Building $\boldsymbol{\Theta}$ as in (26) with $s$ replaced by $\frac{1}{s}$ yields a rational model of the transfer parameters of the filter up to the unrecoverable $\mathbf{T}_{c}$. Since S-parameter data of the filter are available, we can compute this missing factor at each frequency as $\mathbf{T}_{c}\left(\mathrm{i} \omega_{i}\right)=\left(\boldsymbol{\Theta}\left(\frac{1}{i \omega_{i}}\right)\right)^{-1} \mathscr{T}_{\mathbf{U}}\left(\mathbf{S}_{i}\right)$ where $\mathbf{S}_{i}$ is the measured S-parameter measurement at excitation frequency $\omega_{i}$. The mean over all frequencies of the matrix $\mathbf{T}_{c}$ is

$$
\mathbf{T}_{c}=\left[\begin{array}{rr}
16.42-14.81 \mathrm{i} & 7.9-20.98 \mathrm{i} \\
7.3+21.29 \mathrm{i} & 16.51+15.81 \mathrm{i}
\end{array}\right]
$$

and the standard deviation is at most .54 for all entries. Errors inherent to full-wave simulations make the interpolation points and values inexact, leading to a matrix $\mathbf{T}_{c}$ which is not constant over all frequencies, but is not far from it. Comparing the measured S-parameters to those obtained using $\mathbf{T}_{c}$ in (39), we notice a very good match (Fig. 7).

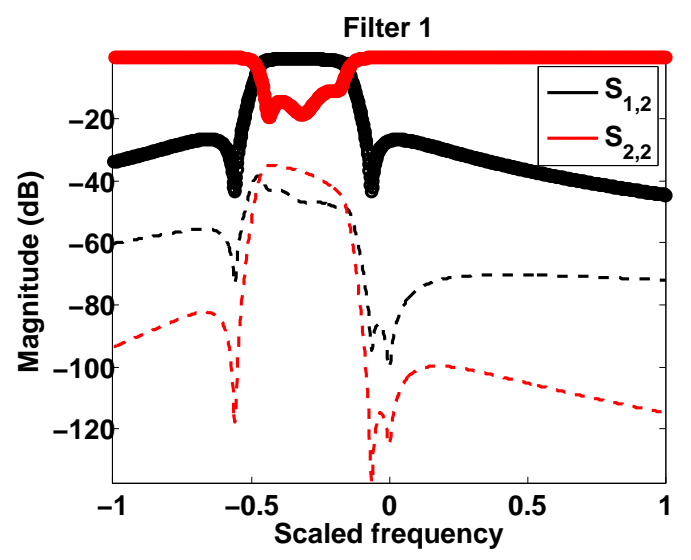

Fig. 7. Measured S-parameters are indistinguishable from those computed with the recovered model. Dashed lines show the errors.

For filter 2, the 4 transmission zeros are identified as the common zeros of the $3^{\text {rd }}$ column of the multi-port model of the triplexer, namely $-.004-.216 \mathrm{i},-.004+.218 \mathrm{i}$ and infinity with multiplicity 2 . The SISO system located on the diagonal entry $(3,3)$ of the triplexer model yields the interpolation values which allow to build $\Theta$ as in (26). The same remark as for filter 1 holds for filter 2 regarding its lossy nature, leading to a missing factor $\mathbf{T}_{c}$ with mean over all frequencies of

$$
\mathbf{T}_{c}=\left[\begin{array}{ll}
0.26+0.92 \mathrm{i} & 0.04-0.15 \mathrm{i} \\
0.04+0.17 \mathrm{i} & 0.29-1.04 \mathrm{i}
\end{array}\right]
$$

and standard deviation of at most .028 for all entries. 
The comparison between the measured and recovered Sparameters is shown in Fig. 8.

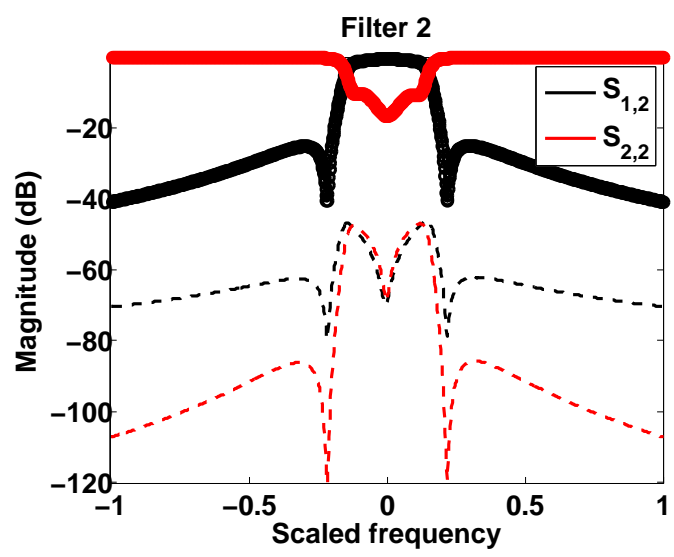

Fig. 8. Measured S-parameters are indistinguishable from those computed with the recovered model. Dashed lines show the errors.

Finally, filter 3 also has two finite transmission zeros at $-.004+.556 \mathrm{i}$ and $-.004+.069 \mathrm{i}$ together with one at infinity with multiplicity 2 . Similarly, due to the lossy nature of the filter, the missing factor $\mathbf{T}_{c}$ is almost constant with mean

$$
\mathbf{T}_{c}=\left[\begin{array}{rr}
-19.9-1.71 \mathrm{i} & 6.3-19.25 \mathrm{i} \\
6.7+19.18 \mathrm{i} & -20.53+2.19 \mathrm{i}
\end{array}\right]
$$

and standard deviation of at most .39 for all entries. The recovered versus the measured S-parameters are shown below.

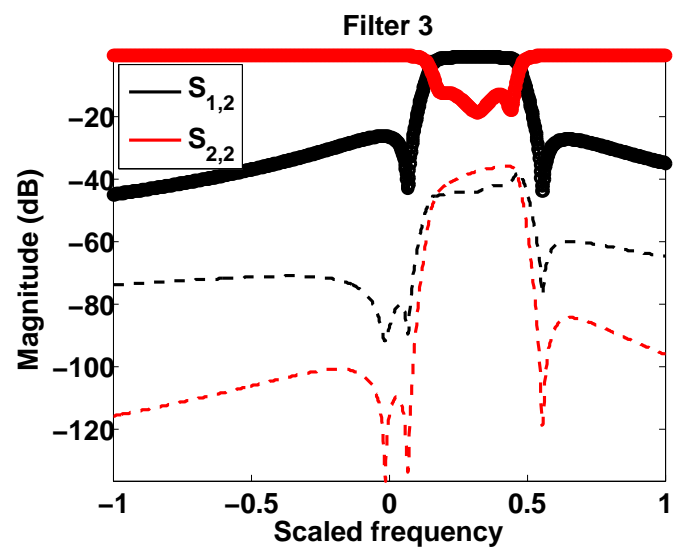

Fig. 9. Measured S-parameters are indistinguishable from those computed with the recovered model. Dashed lines show the errors.

\section{Conclusion}

This paper addresses the de-embedding problem of determining the filters composing a multiplexer from its $S$ parameter measurements by proposing a two-step approach. First a rational model of the whole multiplexer has to be computed from the measurements. Next, starting from this rational model, each filter composing the multiplexer is determined, up to the resonator closest to the junction, by solving a rational interpolation problem, the condition of which are located at the transmission zeros of each filter. Our approach, based on linear factional transformations associated to the interpolation problem, yields all possible solutions of degree $n$. The recovered filters are reciprocal of the right order, but they are not guaranteed passive by the method. The passivity enforcement under minimal degree constraint remains an open and challenging problem of NevanlinnaPick interpolation. Numerical examples demonstrate the effectiveness of the approach, which provides a missing and useful tool for applications in the context of computer assisted tuning and fault diagnosis for multiplexer design.

\section{References}

[1] B.D.O. Anderson and A.C. Antoulas. Rational interpolation and state-variable realizations. Linear Algebra and its Applications, 137138:479-509, 1990.

[2] B.D.O. Anderson and R.W. Newcomb. Cascade connection for timeinvariant n-port networks. Proceedings of the Institution of Electrical Engineers, 113(6):970-974, June 1966.

[3] B.D.O Anderson and V. Vongpanitlerd. Network analysis and synthesis - A modern system theory approach. Prentice Hall, 1973.

[4] A.C. Antoulas and B.D.O. Anderson. On the scalar rational interpolation problem. IMA Journal of Mathematical Control and Information, 3:61-88, 1986.

[5] A.C. Antoulas, J.A. Ball, J. Kang, and J.C. Willems. On the solution of the minimal rational interpolation problem. Linear Algebra and its Applications, 137-138:511-573, 1990.

[6] J.A. Ball, I. Gohberg, and L. Rodman. Interpolation of Rational Matrix Functions, volume 45. Birkhäuser, Basel, 1990.

[7] V. Belevitch. Recent developments in filter theory. IRE Transactions on Circuit Theory, 5(4):236-252, Dec 1958.

[8] C. I. Byrnes, T. T. Georgiou, and A. Lindquist. A generalized entropy criterion for Nevanlinna-Pick interpolation with degree constraint. IEEE Transactions on Automatic Control, 46(6):822-839, Jun 2001.

[9] J. Carlin. Darlington synthesis revisited. IEEE Transactions on Circuits and Systems, 46(1):14-21, 1999.

[10] E.W. Cheney. Introduction to approximation theory. American Mathematical Society, Chelsea, 1982.

[11] H. Dym. J-contractive matrix functions, reproducing kernel Hilbert spaces and interpolation. American Mathematical Society, Rhode Island, 1989.

[12] P. Fulcheri and M. Olivi. Matrix rational $H_{2}$-approximation: a gradient algorithm based on Schur analysis. SIAM Journal on Control and Optimization, 36(6):2103-2127, 1998.

[13] Tryphon T. Georgiou. A topological approach to Nevanlinna-Pick interpolation. SIAM Journal on Mathematical Analysis, 18(5):12481260, 1987.

[14] A. Gombani and G. Michaletzky. On the partial realization problem. In International Symposium on Mathematical Theory of Networks and Systems (MTNS), July 2010.

[15] B. Hanzon, M. Olivi, and R.L.M. Peeters. Balanced realizations of discrete-time stable all-pass systems and the tangential Schur algorithm. Linear Algebra and its Applications, 418(2-3):793-820, Oct. 2006. 
[16] N. Higham. Functions of Matrices: Theory and Computation. Society for Industrial and Applied Mathematics, Philadelphia, 2008.

[17] I. Hunter. Theoy and design of microwave filters. The Institution of Electrical Engineers, London, UK, 2001.

[18] H. Kimura. Chain-Scattering Approach to $H_{\infty}$-Control. Birkhäuser, Basel, 1987.

[19] S. Lefteriu and A.C. Antoulas. A new approach to modeling multiport systems from frequency-domain data. IEEE Trans. Comput.-Aided Design Integr. Circuits Syst., 29(1):14-27, Jan. 2010.

[20] S. Lefteriu, M. Oldoni, M. Olivi, and F. Seyfert. De-embedding multiplexers by Schur reduction. In IEEE 52nd Annual Conference on Decision and Control (CDC), pages 6502-6507, Dec 2013.

[21] A.J. Mayo and A.C. Antoulas. A framework for the solution of the generalized realization problem. Linear Algebra and its Applications, 425(2-3):634-662, Sept. 2007

[22] J.J. Michalski. Artificial neural network algorithm for automated filter tuning with improved efficiency by usage of many golden filters. In 18th International Conference on Microwave Radar and Wireless Communications (MIKON), pages 1-3, June 2010.

[23] M. Olivi, F. Seyfert, and J.-P. Marmorat. Identification of microwave filters by analytic and rational $\mathrm{H}_{2}$ approximation. Automatica, 49(2):317-325, Jan 2013

[24] V. P. Potapov. Linear fractional transformations of matrices (in russian). In V. A. Marcenko, editor, Studies in the Theory of Operators and Their Applications, pages 75-97. Naukova Dumka, Kiev, 1979

[25] F. Seyfert and S. Bila. General synthesis techniques for coupled resonator networks. IEEE Microwave Magazine, 8(5):98-104, 2007.

[26] F. Seyfert, M. Oldoni, M. Olivi, S. Lefteriu, and D. Pacaud. Deembedding of filters in multiplexers via rational approximation and interpolation. In IEEE MTT-S International Microwave Symposium (IMS), pages 1-4, June 2014.

[27] F. Seyfert, M. Oldoni, M. Olivi, S. Lefteriu, and D. Pacaud. Deembedding of filters in multiplexers via rational approximation and interpolation. International Journal of RF and Microwave ComputerAided Engineering, 25(8):647-654, 2015.

[28] D.C. Youla and M. Saito. Interpolation with positive real functions. Journal of the Franklin Institute, 284(2):77-108, 1967.

[29] M. Yu and W. C. Tang. A fully automated filter tuning robots for wireless base station diplexers. In IEEE International Microwave Symposium Workshop Computer Aided Filter Tuning, June 2003.

\section{A Proof of Proposition 3.1}

Applying the product rule in (14) to compute high order derivatives of the multiplexer's S-matrix $\boldsymbol{\Sigma}(s)$, we obtain

$$
\boldsymbol{\Sigma}^{(h)}=\mathbf{S}_{22}^{(h)}+\sum_{i=0}^{h}\left(\begin{array}{c}
h \\
i
\end{array}\right) \sum_{j=0}^{h-i}\left(\begin{array}{c}
h-i \\
j
\end{array}\right) \mathbf{S}_{21}^{(j)} \mathbf{X}^{(h-i-j)} \mathbf{S}_{12}^{(i)}
$$

where the s-dependency of the matrix functions has been omitted and $\mathbf{X}$ stands for $\widetilde{\boldsymbol{\Sigma}}\left(\mathbf{I}_{N+1}-\mathbf{S}_{11} \widetilde{\boldsymbol{\Sigma}}\right)^{-1}$. To prove that $\boldsymbol{\Sigma}^{(h)}(\sigma) \mathbf{e}_{k+1}=S_{k, 22}^{(h)}(\sigma) \mathbf{e}_{k+1}$, for $h=1, \ldots, m-1$, we evaluate (A.1) at $s=\sigma$, multiply by $\mathbf{e}_{k+1}$ on the right and note that $\mathbf{S}_{12}^{(h)}(\sigma) \mathbf{e}_{k+1}=\mathbf{0}$ due to $\sigma$ being a transmission zero of filter $k$ with multiplicity $m$, so derivatives $\mathbf{S}_{k, 12}^{(h)}(\sigma)$ are 0 for $h<m$. The invertibility of $\mathbf{I}_{N+1}-\mathbf{S}_{11} \widetilde{\Sigma}$ at $\sigma$ ensures that no pole-zero cancellation occurs.

Applying the product rule in (14) by grouping $\mathbf{X}$ and $\mathbf{S}_{12}$, the derivative $\boldsymbol{\Sigma}^{(h)}$ can also be expressed as

$\boldsymbol{\Sigma}^{(h)}=\mathbf{S}_{22}^{(h)}+\sum_{i=0}^{h}\left(\begin{array}{c}h \\ i\end{array}\right) \mathbf{S}_{21}^{(i)} \sum_{j=0}^{h-i}\left(\begin{array}{c}h-i \\ j\end{array}\right) \mathbf{X}^{(h-i-j)} \mathbf{S}_{12}^{(j)}$

To show that $\mathbf{e}_{k+1}^{T} \boldsymbol{\Sigma}^{(h)}(\sigma)=\mathbf{e}_{k+1}^{T} S_{k, 22}^{(h)}(\sigma)$, for $h=1, \ldots, m-$ 1 , we evaluate (A.2) for $s=\sigma$, multiply by $\mathbf{e}_{k+1}^{T}$ on the left and note that $\mathbf{e}_{k+1}^{T} \mathbf{S}_{21}^{(h)}(\sigma)=\mathbf{0}$ due to $\sigma$ being a transmission zero of filter $k$ with multiplicity $m$.

Lastly, to show that $\mathbf{e}_{k+1}^{T} \boldsymbol{\Sigma}^{(h)}(\sigma) \mathbf{e}_{k+1}=S_{k, 22}^{(h)}(\sigma)$, for $h=$ $m, \ldots, 2 m-1$, one can use either (A.1) or (A.2) to evaluate at $\sigma$ and multiply by $\mathbf{e}_{k+1}^{T}$ on the left and $\mathbf{e}_{k+1}$ on the right, respectively. Considering (A.2), we obtain

$$
\begin{aligned}
& \mathbf{e}_{k+1}^{T} \boldsymbol{\Sigma}^{(h)}(\sigma) \mathbf{e}_{k+1}=\mathbf{e}_{k+1}^{T} \mathbf{S}_{22}^{(h)}(\sigma) \mathbf{e}_{k+1} \\
& +\sum_{i=0}^{h}\left(\begin{array}{c}
h \\
i
\end{array}\right) \mathbf{e}_{k+1}^{T} \mathbf{S}_{21}^{(i)}(\sigma) \sum_{j=0}^{h-i}\left(\begin{array}{c}
h-i \\
j
\end{array}\right) \mathbf{X}^{(h-i-j)}(\sigma) \mathbf{S}_{12}^{(j)}(\sigma) \mathbf{e}_{k+1}
\end{aligned}
$$

Since $h \geq m$ and $\mathbf{e}_{k+1}^{T} \mathbf{S}_{21}^{(i)}(\sigma)=\mathbf{0}$ for $i \leq m$, we conclude that the first $m$ terms in the double sum are zero, so we have that:

$$
\begin{aligned}
& \mathbf{e}_{k+1}^{T} \boldsymbol{\Sigma}^{(h)}(\sigma) \mathbf{e}_{k+1}=\mathbf{e}_{k+1}^{T} \mathbf{S}_{22}^{(h)}(\sigma) \mathbf{e}_{k+1} \\
& +\sum_{i=m}^{h}\left(\begin{array}{c}
h \\
i
\end{array}\right) \mathbf{e}_{k+1}^{T} \mathbf{S}_{21}^{(i)}(\sigma) \sum_{j=0}^{h-i}\left(\begin{array}{c}
h-i \\
j
\end{array}\right) \mathbf{X}^{(h-i-j)}(\sigma) \mathbf{S}_{12}^{(j)}(\sigma) \mathbf{e}_{k+1}
\end{aligned}
$$

On the other hand, $\mathbf{S}_{12}^{(j)}(\sigma) \mathbf{e}_{k+1}=\mathbf{0}$ because $j \leq m-1$ due to the inequalities: $0 \leq j \leq h-i \leq h-m \leq m-1$. Thus, the double sum is zero, yielding the desired expression.

For the converse, assume that the interpolation conditions in (21) hold simultaneously at some point $\sigma$. We wish to show that $\sigma$ is a transmission zero of multiplicity $m$ for filter $k$. Due to the diagonal structure of $\mathbf{S}_{21}$ and $\mathbf{S}_{22}$, we have that $\Sigma_{j+1, k+1}(s)=S_{j, 21}(s) X_{j+1, k+1}(s) S_{k, 12}(s)$ where $\mathbf{X}$ stands for $\widetilde{\boldsymbol{\Sigma}}\left(\mathbf{I}_{N+1}-\mathbf{S}_{11} \widetilde{\boldsymbol{\Sigma}}\right)^{-1}$ and $j \neq k$. As $\sigma$ is a zero of $\Sigma_{j+1, k+1}$ with multiplicity $\mathrm{m}$, and if we know that it is not a zero of $\mathbf{X}$ (namely, of the junction), neither a zero of all $S_{j, 21}(s)$, for $j=1, \ldots, N, j \neq k$, we conclude that it is a zero of $S_{k, 12}(s)$.

\section{B Proof of Theorem 5.1}

To show the first property, we first derive the following expression of the LFT $H(s)=\mathscr{T}_{\Theta}(\delta)$ :

$$
H(s)=\delta+\left(\mathbf{v}^{T}-\delta \mathbf{u}^{T}\right) \mathbf{X}_{\delta}(s)^{-1}(\mathbf{u} \delta-\mathbf{v}),
$$


where $\mathbf{X}_{\delta}(s)=\mathbb{L}\left(s \mathbf{I}-\mathbf{M}^{T}+\mathbb{L}^{-1}(\mathbf{u} \delta-\mathbf{v}) \mathbf{u}^{T}\right)$ is given by (27). This formula is obtained as in [12, Lemma 9]. Note that $\mathbf{A}=\mathbf{M}^{T}-\mathbb{L}^{-1}(\mathbf{u} \delta-\mathbf{v}) \mathbf{u}^{T}$ is the dynamic matrix of $H(s)$. Since $\mathbf{X}_{\delta}\left(\widetilde{\sigma}_{i}\right)$ is assumed invertible, the determinant of $\widetilde{\sigma}_{i} I-\mathbf{A}$ is not equal to zero and thus $\widetilde{\sigma}_{i}$ is not an eigenvalue of $\mathbf{A}$. Thus, the condition $\lambda(\mathbf{M}) \cap \lambda(\mathbf{A})=\emptyset$ is satisfied.

To check if interpolations conditions hold, we compute

$$
\begin{aligned}
\mathbf{u} H(s)-\mathbf{v} & =\mathbf{u} \delta-\mathbf{v}+\mathbf{u}\left(\mathbf{v}^{T}-\delta \mathbf{u}^{T}\right) \mathbf{X}_{\delta}(s)^{-1}(\mathbf{u} \delta-\mathbf{v}) \\
& \left.=\left[X_{\delta}(s)+\mathbf{u}\left(\mathbf{v}^{T}-\delta \mathbf{u}^{T}\right)\right]\right) \mathbf{X}_{\delta}(s)^{-1}(\mathbf{u} \delta-\mathbf{v}) \\
& =(s \mathbf{I}-\mathbf{M}) \mathbb{L} \mathbf{X}_{\delta}(s)^{-1}(\mathbf{u} \delta-\mathbf{v})
\end{aligned}
$$

In going from (B.3) to (B.4), we used that the matrix $\mathbb{L}$ satisfies the Sylvester equation $\mathbb{L} \mathbf{M}^{T}-\mathbf{M} \mathbb{L}=\mathbf{u v} \mathbf{v}^{T}-\mathbf{v} \mathbf{u}^{T}$ by construction. As $\Gamma$ is any contour which contains the set of interpolation points but no poles of $H(s)$, we have that

$$
\begin{aligned}
& \frac{1}{2 \pi i} \int_{\Gamma}(s \mathbf{I}-\mathbf{M})^{-1}(\mathbf{u} H(s)-\mathbf{v}) d s \\
= & \frac{1}{2 \pi i} \int_{\Gamma} \mathbb{L} \mathbf{X}(s)^{-1}(\mathbf{u} \delta-\mathbf{v})=0 .
\end{aligned}
$$

This proves (23). Moreover, using [6, Lemma 16.10.4] and the matrix Cauchy integral formula [16], we get

$$
\begin{aligned}
& \frac{1}{2 \pi i} \int_{\Gamma}(s \mathbf{I}-\mathbf{M})^{-1} \mathbf{u} H(s) \mathbf{u}^{T}\left(s \mathbf{I}-\mathbf{M}^{T}\right)^{-1} d s \\
= & \frac{1}{2 \pi i} \int_{\Gamma}(s I-\mathbf{M})^{-1}(\mathbf{u} H(s)-v) \mathbf{u}^{T}\left(s I-\mathbf{M}^{T}\right)^{-1} d s \\
= & \frac{1}{2 \pi i} \int_{\Gamma} \mathbb{L} \mathbf{X}(s)^{-1}(\mathbf{u} \in-\mathbf{v}) \mathbf{u}^{T}\left(s \mathbf{I}-\mathbf{M}^{T}\right)^{-1} d s \\
= & \frac{1}{2 \pi i} \int_{\Gamma} \mathbb{L} \mathbf{X}_{\Lambda}(s)^{-1}\left(\mathbf{X}_{\perp}(s)-\mathbb{L}\left(s \mathbf{I}-\mathbf{M}^{T}\right)\right)\left(s \mathbf{I}-\mathbf{M}^{T}\right)^{-1} d s \\
= & -\frac{1}{2 \pi i} \int_{\Gamma} \mathbb{L} \mathbf{X}_{(}(s)^{-1} \mathbb{L} d s+\frac{1}{2 \pi i} \int_{\Gamma} \mathbb{L}\left(s \mathbf{I}-\mathbf{M}^{T}\right)^{-1} d s=\mathbb{L},
\end{aligned}
$$

so the interpolation conditions in (24) are also satisfied.

This completes the proof of the direct statement.

For the converse, first note that, since the interpolation problem has a solution of degree $n$, the Loewner matrix $\mathbb{L}$ must be invertible [4]. We show that the LFT $\mathscr{T}_{\Theta^{-1}}(H(s))$ yields a constant $\delta \in \mathbb{C}$ when starting from a rational proper solution $H(s)$ of degree $n$ to Problem 5.1 with none of its poles coinciding with an interpolation point. We first note that

$$
\boldsymbol{\Theta}^{-1}(s)=\mathbf{I}-\left[\begin{array}{c}
\mathbf{v}^{T} \\
\mathbf{u}^{T}
\end{array}\right] \mathbb{L}^{-1}(s \mathbf{I}-\mathbf{M})^{-1}\left[\begin{array}{ll}
\mathbf{u} & -\mathbf{v}
\end{array}\right]
$$

because $\mathbb{L} \mathbf{M}^{T}-\mathbf{M} \mathbb{L}=\mathbf{u v} \mathbf{v}^{T}-\mathbf{v} \mathbf{u}^{T}$ by construction.

Starting from the minimal state-space realization $H(s)=$ $\mathbf{c}^{T}(s \mathbf{I}-\mathbf{A})^{-1} \mathbf{b}+\delta$ (this $\mathbf{A}, \mathbf{b}, \mathbf{c}$ notation is internal to this proof and is not to be confused with the same notation in Step
4 of the algorithm), the realization of $\widehat{H}(s)=\mathscr{T}_{\Theta^{-1}}(H(s))$ is

$\hat{H}(s)=\left[\begin{array}{c|c}\widehat{\mathbf{A}} & \widehat{\mathbf{b}} \\ \hline \widehat{\mathbf{c}}^{T} & \delta\end{array}\right]=\left[\begin{array}{cc|c}\mathbf{M}+(\delta \mathbf{u}-\mathbf{v}) \mathbf{u}^{T} \mathbb{L}^{-1} & \mathbf{u} \mathbf{c}^{T} & \delta \mathbf{u}-\mathbf{v} \\ \mathbf{b u}^{T} \mathbb{L}^{-1} & \mathbf{A} & \mathbf{b} \\ \hline-\left(\mathbf{v}^{T}-\delta \mathbf{u}^{T}\right) \mathbb{L}^{-1} & \mathbf{c}^{T} & \delta\end{array}\right]$

This is computed using operations on state-space realizations: cascading, inversion and reduction. The realization of $\widehat{H}(s)$ in (B.6) is of size $2 n$. The goal is to show that, in fact, $\widehat{H}(s)$ is constant (hence its McMillan degree is 0 ).

Following [14], we first write the interpolation conditions (23) and (24) for $H(s)$ in state-space form. Let $\mathbf{Y}_{1}, \mathbf{Y}_{2}$ be the unique solutions to

$-\mathbf{M Y} \mathbf{Y}_{1}+\mathbf{Y}_{1} \mathbf{A}+\mathbf{u} \mathbf{c}^{T}=\mathbf{0}, \quad \mathbf{A} \mathbf{Y}_{2}-\mathbf{Y}_{2} \mathbf{M}^{T}+\mathbf{b u} \mathbf{u}^{T}=\mathbf{0}$.

Note that $\left(\mathbf{A}^{T}, \mathbf{c}, \mathbf{b}^{T}, \delta\right)$ being a realization of $H(s)$, there exists a symmetric state-space transformation $\mathbf{T}$ such that $\mathbf{A}^{T}=\mathbf{T A T} \mathbf{T}^{-1}$ and $\mathbf{c}=\mathbf{T b}$ and $\mathbf{Y}_{2}=\mathbf{T}^{-1} \mathbf{Y}_{1}^{T}$. Then, the interpolation vector $\mathbf{v}$ in (23) can be computed as

$$
\mathbf{v}=\mathbf{Y}_{1} \mathbf{b}+\delta \mathbf{u}
$$

In addition, the matrix $\mathbb{L}$ in (24) can be computed as

$$
\mathbb{L}=-\mathbf{Y}_{1} \mathbf{Y}_{2}=-\mathbf{Y}_{1} \mathbf{T}^{-1} \mathbf{Y}_{1}^{T}
$$

First, we reduce the size of the realization from $2 n$ to $n$, so we are looking for a state-space transformation $\breve{\mathbf{T}}$ which allows us to eliminate the uncontrollable states. By choosing

$$
\breve{\mathbf{T}}=\left[\begin{array}{cc}
\mathbf{I} & \mathbf{Y}_{1} \\
\mathbf{0} & \mathbf{I}
\end{array}\right]
$$

we obtain the following equivalent realization

$$
\widehat{H}(s)=\left[\begin{array}{cc|c}
\mathbf{M} & \mathbf{0}_{n \times n} & \mathbf{0}_{1 \times n} \\
\mathbf{b u}^{T} \mathbb{L}^{-1} & \mathbf{A}-\mathbf{b u}^{T} \mathbb{L}^{-1} \mathbf{Y}_{1} & \mathbf{b} \\
\hline-\left(\mathbf{v}^{T}-\delta \mathbf{u}^{T}\right) \mathbb{L}^{-1} & \mathbf{c}^{T}+\left(\mathbf{v}^{T}-\delta \mathbf{u}^{T}\right) \mathbb{L}^{-1} \mathbf{Y}_{1} & \delta
\end{array}\right]
$$

after performing simplifications using (B.7) and (B.8). After eliminating the first $n$ states, which are uncontrollable, we obtain a smaller realization for $\widehat{H}(s)$ :

$$
\widehat{H}(s)=\left[\begin{array}{c|c}
\mathbf{A}-\mathbf{b} \mathbf{u}^{T} \mathbb{L}^{-1} \mathbf{Y}_{1} & \mathbf{b} \\
\hline \mathbf{c}^{T}+\left(\mathbf{v}^{T}-\delta \mathbf{u}^{T}\right) \mathbb{L}^{-1} \mathbf{Y}_{1} & \delta
\end{array}\right] .
$$

Using (B.8) and (B.9), we have that

$\mathbf{c}^{T}+\left(\mathbf{v}^{T}-d \mathbf{u}^{T}\right) \mathbb{L}^{-1} \mathbf{Y}_{1}=\mathbf{c}^{T}+\mathbf{b}^{T} \mathbf{Y}_{1}^{T} \mathbb{L}^{-1} \mathbf{Y}_{1}=\mathbf{c}^{T}-\mathbf{b}^{T} \mathbf{T}=\mathbf{0}$. 
Therefore $\widehat{H}(s)$ is simply $\delta$. The condition $\mathbf{X}_{\delta}\left(\widetilde{\sigma}_{i}\right)$ invertible for $i=0, \ldots, n_{d}$ follows from the assumption on the poles of $H(s)$. 